\title{
NEVANLINNA-PICK INTERPOLATION AND FACTORIZATION OF LINEAR FUNCTIONALS
}

\author{
KENNETH R. DAVIDSON AND RYAN HAMILTON
}

\begin{abstract}
If $\mathfrak{A}$ is a unital weak-* closed algebra of multiplication operators on a reproducing kernel Hilbert space which has the property $\mathbb{A}_{1}(1)$, then the cyclic invariant subspaces index a Nevanlinna-Pick family of kernels. This yields an NP interpolation theorem for a wide class of algebras. In particular, it applies to many function spaces over the unit disk including Bergman space. We also show that the multiplier algebra of a complete NP space has $\mathbb{A}_{1}(1)$, and thus this result applies to all of its subalgebras. A matrix version of this result is also established. It applies, in particular, to all unital weak-* closed subalgebras of $H^{\infty}$ acting on Hardy space or on Bergman space.
\end{abstract}

\section{INTRODUCTION}

The classical interpolation theorem for analytic functions is due to Pick in 1916. Suppose $z_{1}, \ldots, z_{n}$ are distinct points in the complex open disk $\mathbb{D}$ and $w_{1}, \ldots, w_{n}$ are complex numbers. The Nevanlinna-Pick (NP) interpolation theorem asserts that the positivity of the matrix

$$
\left[\frac{1-w_{i} \overline{w_{j}}}{1-z_{i} \overline{z_{j}}}\right]
$$

is a necessary and sufficient condition to ensure the existence of an analytic function $f$ on $\mathbb{D}$ satisfying $f\left(z_{i}\right)=w_{i}$ for $i=1, \ldots, n$ and $\|f\|_{\infty}:=\sup \{|f(z)|: z \in \mathbb{D}\} \leq 1$. Nevanlinna gave a new proof and provided a parameterization of all solutions a few years later.

The seminal work of Sarason [34] reformulated the Nevanlinna-Pick theorem in operator theoretic language. Let $\mathfrak{J}_{E}$ be the ideal of functions in $H^{\infty}(\mathbb{D})$ that vanish on $E=\left\{z_{1}, \ldots, z_{n}\right\}$, and let $M(E)=H^{2} \ominus \overline{\mathfrak{J}_{E} H^{2}}$. Sarason showed that the NP theorem is equivalent to the representation

$$
f+\mathfrak{J}_{E} \mapsto P_{M(E)} M_{f} P_{M(E)}
$$

being isometric, where $M_{f}$ denotes the multiplication operator by $f$ on Hardy space $H^{2}(\mathbb{D})$. He established this using a prototypical version of the commutant lifting theorem. The Szegö kernel $k_{z}^{S}(w)=(1-\bar{z} w)^{-1}$ is the reproducing kernel for $H^{2}(\mathbb{D})$, and one easily sees that $M(E)=\operatorname{span}\left\{k_{z_{1}}^{S}, \ldots, k_{z_{n}}^{S}\right\}$. The operator $P_{M(E)} M_{f}^{*} P_{M(E)}=\left.M_{f}^{*}\right|_{M(E)}$ is diagonal with respect to the (non-orthogonal) basis $k_{\lambda_{1}}^{S}, \ldots, k_{\lambda_{n}}^{S}$ since $M_{f}^{*} k_{z_{i}}^{S}=\overline{f\left(z_{i}\right)} k_{z_{i}}^{S}$. We can summarize the classic NP theorem as

2010 Mathematics Subject Classification. Primary 47A57; Secondary 30E05, 46E22.

Key words and phrases. Nevanlinna-Pick interpolation, reproducing kernel.

First author was partially supported by an NSERC grant.

Second author was partially supported by an NSERC fellowship. 
the distance formula

$$
\operatorname{dist}\left(f, \mathfrak{J}_{E}\right)=\left\|\left.M_{f}^{*}\right|_{M(E)}\right\| .
$$

In [1], Abrahamse proved a Nevanlinna-Pick type theorem on a multiply connected domain $A$. Here, a Pick matrix associated to a single kernel was not sufficient to guarantee the existence of a solution. Instead, an entire family of kernels indexed by copies of the complex torus was required. These spaces arose as subspaces of $L^{2}(\partial A)$ which are rationally invariant. In the case of the annulus, these subspaces were classified by Sarason [33]. Analogous invariant subspaces exist for all nice finitely connected domains. Abrahamse establishes a factorization theorem which shows that certain linear functionals can be represented as rank one functionals on one of these subspaces. This was the first appearance of a Nevanlinna-Pick family of kernels in the literature, and motivated the search for other Nevanlinna-Pick families associated to different algebras of functions.

There is considerable literature concerned with interpolation in the multiplier algebra $\mathfrak{M}(H)$ of a reproducing kernel Hilbert space $H$ over a set $X$. Again, given $E=\left\{\lambda_{1}, \ldots, \lambda_{n}\right\}$ in $X$ and scalars $w_{1}, \ldots, w_{n}$, we are interested in finding a function $f \in \mathfrak{M}(H)$ such that $f\left(\lambda_{i}\right)=w_{i}$ for $1 \leq i \leq n$ and $\left\|M_{f}\right\| \leq 1$. We again define $\mathfrak{J}_{E}=\left\{f \in \mathfrak{M}(H):\left.f\right|_{E}=0\right\}$ and $M(E)=\operatorname{span}\left\{k_{\lambda_{i}}: \lambda_{i} \in E\right\}$. Such a Hilbert space is called a Nevanlinna-Pick kernel if the analogous distance formula holds:

$$
\operatorname{dist}\left(M_{f}, \mathfrak{J}_{E}\right)=\left\|\left.M_{f}^{*}\right|_{M(E)}\right\| .
$$

The kernel functions $k_{\lambda_{i}}$ form a basis for $M(E)$ consisting of eigenvectors for $M_{f}^{*}$, and so the right hand side is at most 1 if and only if the following matrix is positive

$$
\left[\left(1-w_{i} \overline{w_{j}}\right)\left\langle k_{\lambda_{j}}, k_{\lambda_{i}}\right\rangle\right] \text {. }
$$

One can also consider matrix interpolation, and the classical theorem works just as well for matrix algebras over $H^{\infty}$. A kernel for which the classical formula holds for all matrix valued functions is called a complete NP kernel. Results of McCullough 24, 25] and Quiggin [29, 30], building on (unpublished) work by Agler, provide a classification of complete NP kernels. Davidson and Pitts [17] show that the Drury-Arveson space or symmetric Fock space on the unit ball $\mathbb{B}_{d}$ of $\mathbb{C}^{d}$ is a complete NP kernel. Agler and McCarthy [2] showed that every complete NP kernel is equivalent to the restriction of the Drury-Arveson space to some subset of the ball. There is no known characterization of ordinary NP kernels.

In [14], a Nevanlinna-Pick problem on the Hardy space was studied for the subalgebra

$$
H_{1}^{\infty}=\left\{f \in H^{\infty}(\mathbb{D}): f^{\prime}(0)=0\right\} .
$$

Beurling's theorem for the shift was used to characterize the invariant subspace lattice for this algebra. The main result in [14] furnishes a necessary and sufficient condition for the existence of an interpolant in $H_{1}^{\infty}$. This requires the positivity of an entire family of Pick matrices

$$
\left[\left(1-w_{i} \overline{w_{j}}\right)\left\langle P_{L} k_{z_{j}}^{S}, k_{z_{i}}^{S}\right\rangle\right]
$$

for (a family of) invariant subspaces $L$ of $H_{1}^{\infty}$. Again this is equivalent to a distance formula for the ideal $\mathfrak{J}_{E}=\left\{f \in \mathcal{H}_{1}^{\infty}:\left.f\right|_{E}=0\right\}$ :

$$
\operatorname{dist}\left(M_{f}, \mathfrak{J}_{E}\right)=\sup _{L \in \operatorname{Lat}\left(H_{1}^{\infty}\right)}\left\|\left.P_{L \ominus \mathfrak{J}_{E} L} M_{f}^{*}\right|_{L \ominus \mathfrak{J}_{E} L}\right\| .
$$


In this paper, we are interested in general interpolation problems of this type. Consider a reproducing kernel Hilbert space $H$ with kernel $k$ on some set $X$, and a unital weak-*-closed subalgebra $\mathfrak{A}$ of multipliers on $H$. Let $E=\left\{\lambda_{1}, \ldots, \lambda_{n}\right\}$ be a finite subset of points in $X$ which are separated by $\mathfrak{A}$, and let $w_{1}, \ldots, w_{n}$ be complex numbers. We seek a necessary and sufficient condition to ensure the existence of a contractive multiplier in $\mathfrak{A}$ which interpolates the given data. Set $\mathfrak{J}_{E}=\left\{f \in \mathfrak{A}:\left.f\right|_{E}=0\right\}$. It is elementary (see Lemma 2.8) to verify that

$$
\operatorname{dist}\left(M_{f}, \mathfrak{J}_{E}\right) \geq \sup _{L \in \operatorname{Lat} \mathfrak{A}}\left\|\left.P_{L \ominus \mathfrak{J}_{E} L} M_{f}^{*}\right|_{L \ominus \mathfrak{J}_{E} L}\right\| .
$$

Our primary goal here is to find a sufficient condition to ensure that equality holds. When such a formula is satisfied, the algebra $\mathfrak{A}$ is said to have an NP family of kernels. In this case, there is a corresponding family of Pick matrices for which simultaneous positivity of its members is a necessary and sufficient condition for interpolation.

Our main result, Theorem 3.3. states that if $H$ is an reproducing kernel Hilbert space, and $\mathfrak{A}$ is any unital weak-*-closed algebra of multipliers that has the strong predual factorization property called property $\mathbb{A}_{1}(1)$, then $\mathfrak{A}$ admits a NevanlinnaPick family. A weak-* closed subspace of operators $\mathfrak{A}$ has property $\mathbb{A}_{1}(1)$ if every weak-* continuous functional $\varphi$ on $\mathfrak{A}$ with $\|\varphi\|<1$ can be represented as a vector functional $\varphi(A)=\langle A x, y\rangle$ for all $A \in \mathfrak{A}$ with $\|x\|\|y\|<1$. This property was introduced in [19, 4].

While the property $\mathbb{A}_{1}(1)$ is very strong, many relevant examples of multiplier algebras have it. In particular, if the multiplier algebra has $\mathbb{A}_{1}(1)$, then the results apply to all unital weak-* closed subalgebras. A result of Arias and Popescu [5] shows that quotients of the non-commutaitve Toeplitz algebra have property $\mathbb{A}_{1}(1)$. We use this to show that the multiplier algebras of all complete NP spaces have $\mathbb{A}_{1}(1)$. Thus these results apply in many well known contexts including all subalgebras of the multipliers on Drury-Arveson space. Theorem 3.3 provides an NP theorem for any weak-*-closed subalgebra of $H^{\infty}$ acting on Hardy space. This provides a generalization of the results in [14] and [31]. In [32], Raghupathi shows that Abrahamse's interpolation theorem is equivalent to a constrained interpolation problem on certain weak-*-closed subalgebras of $H^{\infty}$. Consequently our distance formula implies Abrahamse's result as well.

Additionally, the above distance formula is still achieved by restricting to the cyclic invariant subspaces of $\mathfrak{A}$. In certain cases of interest, it suffices to use cyclic vectors which do not vanish on $E$. In the case of Hardy space and Drury-Arveson space, this is accomplished by showing that cyclic subspaces generated by outer functions suffice. This yields a simplification in the statement of the theorems.

The distance formula also formulates the classic Nevanlinna-Pick problem in terms of the Bergman kernel, whose multiplier algebra has a property much stronger than $\mathbb{A}_{1}(1)$. Indeed, Bergman space satisfies even a complete distance formula; see Section 7 While only of theoretical interest, since interpolation problems for $H^{\infty}$ are better carried out on Hardy space, this result is surprising since Bergman space is not an NP kernel, and this failure persists even for 2-point interpolation.

In Section 5, algebras of multipliers on complete Nevanlinna-Pick spaces will be studied. The multipliers of any such space are complete quotients of the noncommutative analytic Toeplitz algebra $\mathfrak{L}_{d}$ [17], which has the property $\mathbb{A}_{1}(1)[\mathbf{1 6}$ ] and even the much stronger property $X_{0,1}$ [12]. In the proof of Theorem 5.2, this is 
used to show that all complete NP kernel multiplier algebras inherit $\mathbb{A}_{1}(1)$. Consequently, any weak-*closed algebra of multipliers on a complete NP space admits an NP family of kernels.

Finally, in Section 7 matrix-valued Nevanlinna-Pick problems are studied. In order to retrieve a suitable NP theorem for matrices of arbitrary size, something much stronger than $\mathbb{A}_{1}(1)$ is required. However, by working with a suitable ampliation of the algebra, it is possible to retrieve an NP-type theorem with milder assumptions. Both of these results are summarized in Theorem 7.2 From this, a complete NP family of kernels is presented for the Bergman space as well as the classic matrix-valued NP theorem. A recent result of Ball, Bolotnikov and Ter Horst [7] regarding matrix-valued interpolation on the algebra $H_{1}^{\infty}$ is generalized to arbitrary unital weak-* closed subalgebras of $H^{\infty}$.

\section{NeVAnLinna-PiCK FAmilies}

Let $H$ denote a reproducing kernel Hilbert space of complex-valued functions on a set $X$, with $k_{\lambda}$ as the reproducing kernel at $\lambda \in X$. Let $k(\lambda, \mu):=\left\langle k_{\mu}, k_{\lambda}\right\rangle$ denote the associated positive kernel on $X \times X$. We define the multiplier algebra of $H$ by

$$
\mathfrak{M}(H)=\{f: X \rightarrow \mathbb{C}: f h \in H \text { for all } h \in H\},
$$

where the product $f g$ is defined pointwise. Each multiplier $f$ on $H$ defines the corresponding multiplication operator $M_{f}$, which is bounded by an application of the closed graph theorem. We may then regard $\mathfrak{M}(H)$ as an abelian subalgebra of $\mathcal{B}(H)$. It is well known that an operator $T \in \mathcal{B}(H)$ defines a multiplication operator on $H$ if and only if each kernel function $k_{\lambda}$ is an eigenvector for $T^{*}$. From this it easily follows that $\mathfrak{M}(H)$ is closed in the weak operator topology.

We say that a unital subalgebra $\mathfrak{A}$ of $\mathcal{B}(H)$ is a dual algebra if it is closed in the weak-* topology on $\mathcal{B}(H)$. If $\mathfrak{A}$ is contained in the multiplier algebra of $H$, we say that $\mathfrak{A}$ is a dual algebra of multipliers of $H$. Suppose that $E$ is a finite subset of $X$. Let $\mathfrak{J}_{E}$ denote the weak-* closed ideal of multipliers in $\mathfrak{A}$ that vanish on $E$. When $E=\{\lambda\}$, we write $\mathfrak{J}_{\lambda}$. If the context is clear, we may write $\mathfrak{J}:=\mathfrak{J}_{E}$.

Suppose $L$ is an invariant subspace of $\mathfrak{A}$ and let $P_{L}$ denote the orthogonal projection of $H$ onto $L$. Then $L$ is also a reproducing kernel Hilbert space on the points in $X$ which are not annihilated by $L$. The reproducing kernel on this set is given by $P_{L} k_{\lambda}$. Every $f \in \mathfrak{A}$ defines a multiplier on $L$ since

$$
\left(\left.M_{f}\right|_{L}\right)^{*} P_{L} k_{\lambda}=P_{L} M_{f}^{*} P_{L} k_{\lambda}=P_{L} M_{f}^{*} k_{\lambda}=\overline{f(\lambda)} P_{L} k_{\lambda}
$$

The following lemma shows that, in certain cases, it is possible to extend this kernel to all of $X$.

Lemma 2.1. Suppose $\mathfrak{A}$ is a dual algebra of multipliers on $H$. If $L=\mathfrak{A}[h]$ is a cyclic invariant subspace of $\mathfrak{A}$, it is possible to extend the kernel function $P_{L} k_{\lambda}$ (which is non-zero on $\{\lambda: h(\lambda) \neq 0\}$ ) to a kernel $k_{\lambda}^{L}$ defined on all of $X$ so that $k_{\lambda}^{L}=0$ only when $\mathfrak{J}_{\lambda}[h]=\mathfrak{A}[h]$. For each $f \in \mathfrak{M}(H)$, $k_{\lambda}^{L}$ satisfies the fundamental relation

$$
P_{L} M_{f}^{*} k_{\lambda}^{L}=\overline{f(\lambda)} k_{\lambda}^{L}
$$

and thus

$$
\left\langle M_{f} k_{\lambda}^{L}, k_{\lambda}^{L}\right\rangle=f(\lambda)\left\|k_{\lambda}^{L}\right\|^{2} \quad \text { for all } \quad \lambda \in X
$$


Proof. Since $L=\mathfrak{A}[h]$ is a cyclic subspace and $\operatorname{dim} \mathfrak{A} / \mathfrak{J}_{\lambda}=1$, it follows that $\operatorname{dim} \mathfrak{A}[h] / \mathfrak{J}_{\lambda}[h] \leq 1$. If $P_{L} k_{\lambda} \neq 0$, then this is an eigenvector for $P_{L} \mathfrak{A}^{*}$ as shown above. For $f \in \mathfrak{J}_{\lambda}$,

$$
\left\langle k_{\lambda}^{L}, f h\right\rangle=\left\langle P_{L} M_{f}^{*} k_{\lambda}^{L}, h\right\rangle=\left\langle\overline{f(\lambda)} k_{\lambda}^{L}, h\right\rangle=0 .
$$

So $P_{L} k_{\lambda}$ belongs to $\mathfrak{A}[h] \ominus \mathfrak{J}_{\lambda}[h]$ and we set $k_{\lambda}^{L}=P_{L} k_{\lambda}$.

When $P_{L} k_{\lambda}=0$ but $\operatorname{dim} \mathfrak{A}[h] / \mathfrak{J}_{\lambda}[h]=1$, we pick a unit vector $k_{\lambda}^{L}$ in $\operatorname{dim} \mathfrak{A}[h] \ominus$ $\mathfrak{J}_{\lambda}[h]$. Then for $f \in \mathfrak{M}(H), f-f(\lambda) \mathbf{1}$ lies in $\mathfrak{J}_{\lambda}$. Hence

$$
\left\langle M_{f} k_{\lambda}^{L}, k_{\lambda}^{L}\right\rangle=f(\lambda)\left\langle k_{\lambda}^{L}, k_{\lambda}^{L}\right\rangle-\left\langle(f-f(\lambda) \mathbf{1}) k_{\lambda}^{L}, k_{\lambda}^{L}\right\rangle=f(\lambda) .
$$

Also, $\mathfrak{J}_{\lambda}[h] \in \operatorname{Lat}(\mathfrak{A})$ and thus $P_{L} M_{f}^{*} k_{\lambda}^{L}$ lies in $L \ominus \mathfrak{J}_{\lambda}[h]=\mathbb{C} k_{\lambda}^{L}$. The previous computation shows that

$$
P_{L} M_{f}^{*} k_{\lambda}^{L}=\overline{f(\lambda)} k_{\lambda}^{L} .
$$

This extends the kernel $k^{L}$ to all of $X$.

This kernel allows us to evaluate the multipliers at a generally much larger subset of $X$ (see Example 2.3 below) than just using $P_{L} k_{\lambda}$. However some continuity is lost for evaluation of functions in $L$. Since we are primarily interested in interpolation questions about the multiplier algebra, evaluation of the multipliers is more important.

Definition 2.2. For any dual algebra $\mathfrak{A}$ of multipliers on $H$ and any cyclic invariant subspace $L \in \operatorname{CycLat}(\mathfrak{A})$, let $k_{\lambda}^{L}$ denote the extended reproducing kernel on $L$ constructed in Lemma 2.1 at the point $\lambda$.

Example 2.3. In spaces of analytic functions, it is often possible to fully describe the kernel structure on $L \in \operatorname{CycLat}(\mathfrak{A})$. Indeed, suppose that $H=A^{2}(\mathbb{D})$ is the Bergman space, and $\mathfrak{A}=H^{\infty}$. Let $L=H^{\infty}[h]$ for some non-zero function $h \in A^{2}(\mathbb{D})$. Then

$$
X_{L}=\left\{\lambda \in \mathbb{D}: P_{L} k_{\lambda} \neq 0\right\}=\{\lambda \in \mathbb{D}: h(\lambda) \neq 0\} .
$$

However, since the Bergman space consists of analytic functions, $h$ vanishes only to some finite order on each of its zeros.

It is routine to verify that for each $n \geq 0$ and $\lambda \in \mathbb{D}$, there is a function $k_{\lambda, n} \in A^{2}(\mathbb{D})$ such that

$$
\left\langle h, k_{\lambda, n}\right\rangle=h^{(n)}(\lambda) \quad \text { for } \quad h \in A^{2}(\mathbb{D}) .
$$

Suppose that $h$ vanishes at $\lambda$ with multiplicity $r \geq 0$. We claim that $P_{L} k_{\lambda, r} \neq 0$ and $P_{L} k_{\lambda, n}=0$ for $0 \leq n<r$. Indeed, for any $f \in H^{\infty}$ and $n \leq r$,

$$
\begin{aligned}
\left\langle f h, k_{\lambda, n}\right\rangle & =(f h)^{(r)}\left(\lambda_{i}\right) \\
& =\sum_{j=0}^{r}\left(\begin{array}{l}
r \\
j
\end{array}\right) f^{(j)}(\lambda) h^{(r-j)}(\lambda) \\
& =\left\{\begin{array}{lll}
0 & \text { if } & 0 \leq n<r \\
f(\lambda) h^{(r)}(\lambda) & \text { if } & n=r .
\end{array}\right.
\end{aligned}
$$


So $P_{L} k_{\lambda, n}=0$ for $0 \leq n<r$. Set $k_{\lambda}^{L}=P_{L} k_{\lambda, r} /\left\|P_{L} k_{\lambda, r}\right\|$. This calculation shows that if $f \in \mathfrak{J}_{\lambda}$, then $\left\langle\bar{f} h, k_{\lambda}^{L}\right\rangle=0$. So $k_{\lambda}^{L}$ belongs to $\mathfrak{A}[h] \ominus \mathfrak{J}_{\lambda}[h]$. Now for $f, g \in H^{\infty}$,

$$
\begin{aligned}
\left\langle f h, M_{g}^{*} k_{\lambda}^{L}\right\rangle & =\left\langle g f h, k_{\lambda}^{L}\right\rangle \\
& =g(\lambda) f(\lambda) h^{(r)}\left(\lambda_{i}\right)=\left\langle f h, \overline{g(\lambda)} k_{\lambda}^{L}\right\rangle .
\end{aligned}
$$

It follows that

$$
\left(\left.M_{g}\right|_{L}\right)^{*} k_{\lambda}^{L}=P_{L} M_{g}^{*} k_{\lambda}^{L}=\overline{g(\lambda)} k_{\lambda}^{L} .
$$

Thus $g$ is a multiplier for this reproducing kernel.

An identical construction is possible for any space of analytic functions on the unit disk in which the Taylor coefficients of the power series expansion about 0 are continuous and composition by the Möbius automorphisms of the disk are bounded maps.

Remark 2.4. The Bergman space is also a good place to illustrate why we require cyclic invariant subspaces. The Bergman shift $B$ is a universal dilator for strict contractions [4]. For example, fix a point $\lambda \in \mathbb{D}$. Then $B$ has an invariant subspace $L$ such that $N=L \ominus \mathfrak{J}_{\lambda} L$ is infinite dimensional. Since $\left.\left(\left.M_{f}\right|_{L}\right)^{*}\right|_{N}=\overline{f(\lambda)} I_{N}$, there is no canonical choice for $k_{\lambda}^{L}$. Similarly, for any $0<r<1$, we can obtain the infinite ampliation $r B^{(\infty)}$ as the compression of $B$ to a semi-invariant subspace $M$, and this has the same issue for every $|\lambda|<r$.

On the other hand, we can always identify a kernel structure on any invariant subspace $L \in$ Lat $\mathfrak{A}$ if we allow multiplicity. The subspaces $N_{\lambda}=L \ominus \mathfrak{J}_{\lambda} L$ satisfy $\left.P_{N_{\lambda}} M_{f}^{*}\right|_{N_{\lambda}}=\overline{f(\lambda)} P_{N_{\lambda}}$. So if $k$ is any unit vector in $N_{L}$, we obtain

$$
\left\langle M_{f} k, k\right\rangle=f(\lambda) \text { for all } f \in \mathfrak{A} .
$$

The spaces $\left\{N_{\lambda}: \lambda \in X\right\}$ are linearly independent and together they span $L$. See the continued discussion later in Remark 2.9.

Our primary interest will be Nevanlinna-Pick interpolation on some finite subset $E=\left\{\lambda_{1}, \ldots, \lambda_{n}\right)$ of $X$ by functions in the algebra $\mathfrak{A}$. It could be the case that $\mathfrak{A}$ fails to separate certain points in $X$, and so we impose the natural constraint that $E$ contains at most one representative from any set of points that $\mathfrak{A}$ identifies. It follows that the kernels $k_{\lambda_{i}}$ form a linearly independent set. Indeed, since $\mathfrak{A}$ separates these points, we can find elements $p_{1}, \ldots, p_{n} \in \mathfrak{A}$ such that $p_{i}\left(\lambda_{j}\right)=\delta_{i j}$. Hence if $\sum_{i=1}^{n} \alpha_{i} k_{\lambda_{i}}=0$, we find that

$$
0=M_{p_{i}}^{*}\left(\sum_{i=1}^{n} \alpha_{i} k_{\lambda_{i}}\right)=\alpha_{i} k_{\lambda_{i}} \quad \text { for } \quad 1 \leq i \leq n .
$$

The quotient algebra $\mathfrak{A} / \mathfrak{J}_{E}$ is $n$-dimensional, and is spanned by the idempotents $\left\{p_{i}+\mathfrak{J}: 1 \leq i \leq n\right\}$. We seek to establish useful formulae for the norm on $\mathfrak{A} / \mathfrak{J}$. These so-called operator algebras of idempotents have been studied by Paulsen in [27].

Definition 2.5. Given a finite subset $E$ of $X$, set

$$
M=M(E)=\operatorname{span}\left\{k_{\lambda}: \lambda \in E\right\} .
$$

For $L \in \operatorname{Lat}(\mathfrak{A})$, define

$$
M_{L}=P_{L} M \quad \text { and } \quad N_{L}=L \ominus \mathfrak{J}_{E} L .
$$


Observe that if $L \in$ Lat $\mathfrak{A}$, then $\overline{\mathfrak{J}_{E} L}$ is also invariant and is contained in $L$. Thus $N_{L}$ is a semi-invariant subspace. In particular, $P_{N_{L}} M_{f} P_{L}=P_{N_{L}} M_{f} P_{N_{L}}$ and compression to $N_{L}$ is a contractive homomorphism. Likewise, $M(E)$ is co-invariant, and so $\overline{M(E)+L^{\perp}}$ is co-invariant. Observe that

$$
M_{L}=\overline{\left(M(E)+L^{\perp}\right)} \cap L=L \ominus\left(M(E)+L^{\perp}\right)^{\perp},
$$

and so it is also semi-invariant.

Lemma 2.6. Given a finite subset $E \subset X$ on which $\mathfrak{A}$ separates points, and $L=$ $\mathfrak{A}[h]$ in CycLat $(\mathfrak{A})$, the space $N_{L}$ is a reproducing kernel Hilbert space over $E$ with kernel $\left\{k_{\lambda}^{L}: \lambda \in E\right\}$; and the non-zero elements of this set form a basis for $N_{L}$.

Also $M_{L}$ is a subspace of $N_{L}$ spanned by

$$
\left\{k_{\lambda}^{L}=P_{L} k_{\lambda}: \lambda \in E, h(\lambda) \neq 0\right\},
$$

and it is a reproducing kernel Hilbert space over $\{\lambda \in E: h(\lambda) \neq 0\}$.

Proof. For each $\lambda \in E$,

$$
k_{\lambda}^{L} \in L \ominus \mathfrak{J}_{\lambda}[h] \subset L \ominus \mathfrak{J}_{E}[h]=N_{L} .
$$

Let $E_{L}=\left\{\lambda \in E: k_{\lambda}^{L} \neq 0\right\}$. Then for $\lambda \in E \backslash E_{L}, L=\overline{\mathfrak{J}_{\lambda} L}$. Since $\mathfrak{J}_{E_{L}}=$ $\prod_{\lambda \in E \backslash E_{L}} \mathfrak{J}_{\lambda}$, we see that $\overline{\mathfrak{J}_{E_{L}} L}=L$. Hence we may factor $\mathfrak{J}_{E}=\mathfrak{J}_{E_{L}} \mathfrak{J}_{E \backslash E_{L}}$ and note that

$$
\overline{\mathfrak{J}_{E} L}=\overline{\mathfrak{J}_{E_{L}} \overline{\mathfrak{J}_{E \backslash E} L}}=\overline{\mathfrak{J}_{E_{L}} L} .
$$

Now $\operatorname{dim} \mathfrak{A} / \mathfrak{J}_{E_{L}}=\left|E_{L}\right|$, so $\operatorname{dim} N_{L} \leq\left|E_{L}\right|$. But $N_{L}$ contains the non-zero vectors $k_{\lambda}^{L}$ for $\lambda \in E_{L}$. For $f \in \mathfrak{A}$ and $\lambda \in E_{L}$,

$$
P_{N_{L}} M_{f}^{*} k_{\lambda}^{L}=P_{L} P_{\mathfrak{J}_{E}[h]}^{\perp} M_{f}^{*} k_{\lambda}^{L}=P_{L} M_{f}^{*} k_{\lambda}^{L}=\overline{f(\lambda)} k_{\lambda}^{L} .
$$

Because $\mathfrak{A}$ separates the points of $E_{L}$, it follows that these vectors are eigenfunctions for distinct characters of $\mathfrak{A}$, and thus are linearly independent. This set has the same cardinality as $\operatorname{dim} N_{L}$, and therefore it forms a basis.

Now $M_{L}$ is spanned by $\left\{P_{L} k^{\lambda}: \lambda \in E\right\}$, and it suffices to use the non-zero elements. These coincide with $k_{\lambda}^{L}$ on $E_{L}^{0}:=\{\lambda \in E: h(\lambda) \neq 0\}$. This is a subset of the basis for $N_{L}$, and hence $M_{L}$ is a subspace of $N_{L}$. It is also a reproducing kernel space on $E_{L}^{0}$.

The equality $M_{L}=N_{L}$ holds in the following important case. The proof is immediate from the lemma.

Corollary 2.7. Suppose that $E$ is a finite subset of $X$ on which $\mathfrak{A}$ separates points, and $L=\mathfrak{A}[h]$ in CycLat $(\mathfrak{A})$. If $h$ does not vanish on $E$, then $M_{L}=N_{L}$.

We will be searching for appropriate distance formulae from elements of $\mathfrak{A}$ to an ideal $\mathfrak{J}_{E}$ in order to obtain Nevanlinna-Pick type results. We note certain easy estimates which always hold.

Lemma 2.8. Suppose that $\mathfrak{A}$ is a dual algebra on $H$, and let $\mathfrak{J}$ be a wOT-closed ideal. Set $N_{L}=L \ominus \mathfrak{J} L$ and $M_{L}=P_{L} M(E)$ for $L \in$ Lat $\mathfrak{A}$. Then the following distance estimates hold:

$$
\begin{aligned}
\operatorname{dist}(f, \mathfrak{J}) & \geq \sup _{L \in \operatorname{Lat}(\mathfrak{A})}\left\|P_{N_{L}} M_{f} P_{N_{L}}\right\|=\sup _{L \in \operatorname{CycLat}(\mathfrak{A})}\left\|P_{N_{L}} M_{f} P_{N_{L}}\right\| \\
& \geq \sup _{L \in \operatorname{CycLat}(\mathfrak{A})}\left\|P_{M_{L}} M_{f} P_{M_{L}}\right\|=\sup _{L \in \operatorname{Lat}(\mathfrak{A})}\left\|P_{M_{L}} M_{f} P_{M_{L}}\right\| .
\end{aligned}
$$


Proof. Suppose $L$ is an invariant subspace of $\mathfrak{A}$. For $M_{f} \in \mathfrak{A}$ and $M_{g} \in \mathfrak{J}$, compute

$$
\begin{aligned}
\left\|M_{f}-M_{g}\right\| & \geq\left\|P_{N_{L}}\left(M_{f}-M_{g}\right) P_{N_{L}}\right\| \\
& =\left\|P_{N_{L}} M_{f} P_{N_{L}}\right\| \geq\left\|P_{M_{L}} M_{f} P_{M_{L}}\right\| .
\end{aligned}
$$

Taking an infimum over $g \in \mathfrak{J}$ and a supremum over Lat( $\mathfrak{A})$, we obtain

$$
\operatorname{dist}(f, \mathfrak{J}) \geq \sup _{L \in \operatorname{Lat}(\mathfrak{A})}\left\|P_{N_{L}} M_{f} P_{N_{L}}\right\| \geq \sup _{L \in \operatorname{CycLat}(\mathfrak{A})}\left\|P_{N_{L}} M_{f} P_{N_{L}}\right\| .
$$

Since $M_{L}$ is contained in $N_{L}$, we have

$$
\sup _{L \in \operatorname{CycLat}(\mathfrak{A})}\left\|P_{N_{L}} M_{f} P_{N_{L}}\right\| \geq \sup _{L \in \operatorname{CycLat}(\mathfrak{A})}\left\|P_{M_{L}} M_{f} P_{M_{L}}\right\| .
$$

Now consider an arbitrary element $L \in \operatorname{Lat}(\mathfrak{A})$. Then

$$
\begin{aligned}
\left\|P_{N_{L}} M_{f} P_{N_{L}}\right\| & =\left\|P_{N_{L}} M_{f} P_{L}\right\|=\sup _{\|h\|=1, h \in L}\left\|P_{N_{L}} M_{f} P_{L} h\right\| \\
& =\sup _{\|h\|=1, h \in L}\left\|\left(P_{L}-P_{\mathfrak{J}_{E} L}\right) M_{f} P_{\mathfrak{A}[h]} h\right\| \\
& \leq \sup _{\|h\|=1, h \in L}\left\|\left(P_{L}-P_{\mathfrak{J}_{E} \mathfrak{A}[h]}\right) P_{N_{\mathfrak{A}[h]}} M_{f} P_{\mathfrak{A}[h]} h\right\| \\
& =\sup _{\|h\|=1, h \in L}\left\|P_{N_{\mathfrak{Q}[[h]}} M_{f} P_{\mathfrak{A}[h]} h\right\| \\
& \leq \sup _{L \in \operatorname{CycLat}(\mathfrak{A})}\left\|P_{N_{L}} M_{f} P_{L}\right\|=\sup _{L \in \operatorname{CycLat}(\mathfrak{d})}\left\|P_{N_{L}} M_{f} P_{N_{L}}\right\| .
\end{aligned}
$$

Similarly,

$$
\begin{aligned}
\left\|P_{M_{L}} M_{f} P_{M_{L}}\right\| & =\left\|P_{M_{L}} M_{f} P_{L}\right\|=\sup _{\|h\|=1, h \in L}\left\|P_{M_{L}} M_{f} P_{L} h\right\| \\
& =\sup _{\|h\|=1, h \in L}\left\|P_{M_{L}} M_{f} P_{\mathfrak{A}[h]} h\right\| \\
& =\sup _{\|h\|=1, h \in L}\left\|P_{M_{L}} P_{N_{\mathfrak{A}[h]}} M_{f} P_{\mathfrak{A}[h]} h\right\| \\
& =\sup _{\|h\|=1, h \in L}\left\|P_{M_{\mathfrak{A}[h]}} M_{f} P_{\mathfrak{A}[h]} h\right\| \\
& \leq \sup _{L \in \operatorname{CycLat}(\mathfrak{A})}\left\|P_{M_{L}} M_{f} P_{L}\right\|=\sup _{L \in \operatorname{CycLat}(\mathfrak{A})}\left\|P_{M_{L}} M_{f} P_{M_{L}}\right\| .
\end{aligned}
$$

So we may take the supremum over all invariant subspaces without changing these two distance estimates.

When $\mathfrak{A}$ is a dual algebra of multipliers, it is convenient to work with the adjoints due to their rich collection of eigenvectors. Lemma 2.8 gives us

$$
\operatorname{dist}(f, \mathfrak{J}) \geq \sup _{L \in \operatorname{Lat}(\mathfrak{A})}\left\|\left.P_{L} M_{f}^{*}\right|_{N_{L}}\right\|=\sup _{L \in \operatorname{CycLat}(\mathfrak{A})}\left\|\left.P_{N_{L}} M_{f}^{*}\right|_{N_{L}}\right\| .
$$

Remark 2.9. This proposition shows that it suffices to look at cyclic subspaces. In view of Lemma 2.1 this is of particular importance when dealing with algebras of multipliers. But in fact, there is little additional complication when the subspaces $N_{\lambda}=L \ominus \mathfrak{J}_{\lambda} L$ have dimension greater than one.

These subspaces are at a positive angle to each other even when they are infinite dimensional because the restriction of $P_{L} M_{f}^{*}$ to $N_{\lambda}$ is just $\overline{f(\lambda)} P_{N_{\lambda}}$. When $\mathfrak{A}$ separates points $\lambda$ and $\mu$, the boundedness of $M_{f}^{*}$ yields a positive angle between eigenspaces. Moreover the spaces $N_{\lambda}$ for $\lambda \in X$ span $L$. 
We are interested in the norm $\left\|\left.P_{N_{L}} M_{f}^{*}\right|_{N_{L}}\right\|$. This is approximately achieved at a vector $h=\sum x_{\lambda}$ where this is a finite sum of vectors $x_{\lambda} \in N_{\lambda}$. Since

$$
P_{L} M_{f}^{*} h=\sum \overline{f(\lambda)} x_{\lambda},
$$

it follows that $K=\operatorname{span}\left\{x_{\lambda}: \lambda \in X\right\}$ is invariant for $P_{L} M_{f}^{*}$ for all $M_{f} \in \mathfrak{A}$. In particular, we obtain that $\left\|\left.P_{N_{L}} M_{f}^{*}\right|_{N_{L}}\right\| \leq 1$ if and only if $\left\|\left.P_{K} M_{f}^{*}\right|_{K}\right\| \leq 1$ for each subspace $K$ of the form just described. This is equivalent to saying $P_{K}-$ $\left.P_{K} M_{f} M_{f}^{*}\right|_{K} \geq 0$ because of semi-invariance. Because the $x_{\lambda}$ span $K$, this occurs if and only if

$$
0 \leq\left[\left\langle\left(I-M_{f} M_{f}^{*}\right) x_{\lambda_{j}}, x_{\lambda_{i}}\right\rangle\right]=\left[\left(1-f\left(\lambda_{i}\right) \overline{f\left(\lambda_{j}\right)}\right)\left\langle x_{\lambda_{j}}, x_{\lambda_{i}}\right\rangle\right] .
$$

Thus, the norm condition is equivalent to the simultaneous positivity of a family of Pick matrices.

Moreover, in the case of $\mathfrak{J}=\mathfrak{J}_{E}$ for a finite set $E=\left\{\lambda_{1}, \ldots, \lambda_{n}\right\}$ which is separated by $\mathfrak{A}$, this family of Pick matrices is positive if and only if we have positivity of the operator matrix

$$
\left[\left(1-f\left(\lambda_{i}\right) \overline{f\left(\lambda_{j}\right)}\right) P_{N_{\lambda_{i}}} P_{N_{\lambda_{j}}}\right]_{n \times n} .
$$

For an arbitrary ideal $\mathfrak{J}$, we can take the supremum over all finite subsets $E$ of $X$.

Definition 2.10. The collection $\left\{k^{L}: L \in \operatorname{CycLat}(\mathfrak{A})\right\}$ is said to be a NevanlinnaPick family of kernels for $\mathfrak{A}$ if for every finite subset $E$ of $X$ and every $f \in \mathfrak{A}$, (and $\left.N_{L}=L \ominus \mathfrak{J}_{E} L\right)$

$$
\operatorname{dist}\left(f, \mathfrak{J}_{E}\right)=\sup _{L \in \operatorname{CycLat}(\mathfrak{A})}\left\|\left.P_{N_{L}} M_{f}^{*}\right|_{N_{L}}\right\| .
$$

The following routine theorem reveals why Nevanlinna-Pick families are given their name.

Theorem 2.11. Let $\mathfrak{A}$ be a dual algebra of multipliers on a Hilbert space $H$. The family $\left\{k^{L}: L \in\right.$ CycLat $\left.(\mathfrak{A})\right\}$ is a Nevanlinna-Pick family of kernels for $\mathfrak{A}$ if and only if the following statement holds:

Given $E=\left\{\lambda_{1}, \ldots, \lambda_{n}\right\}$ distinct points in $X$ which are separated by $\mathfrak{A}$, and complex scalars $w_{1}, \ldots, w_{n}$, there is a multiplier $f$ in the unit ball of $\mathfrak{A}$ such that $f\left(\lambda_{i}\right)=w_{i}$ for $1 \leq i \leq n$ if and only if the Pick matrices

$$
\left[\left(1-w_{i} \overline{w_{j}}\right) k^{L}\left(\lambda_{i}, \lambda_{j}\right)\right]_{n \times n}
$$

are positive definite for every $L \in \operatorname{CycLat}(\mathfrak{A})$.

Proof. Suppose that $\left\{k^{L}: L \in \operatorname{CycLat}(\mathfrak{A})\right\}$ is a Nevalinna-Pick family. If such a multiplier $f$ exists, then the positivity of the matrices follows from standard results in reproducing kernel Hilbert spaces (see [3], for instance).

On the other hand, suppose that all such matrices are positive definite. Since $\mathfrak{A}$ separates the points in $E$, find an arbitrary interpolant $p \in \mathfrak{A}$ so that $p\left(\lambda_{i}\right)=w_{i}$ (for example, consider the functions $p_{i}$ as defined earlier, and let $p=\sum_{i=1}^{n} w_{i} p_{i}$ ). It is a routine argument in this theory that $\left\|\left.P_{N_{L}} M_{p}^{*}\right|_{N_{L}}\right\| \leq 1$ if and only if

$$
I-P_{N_{L}} M_{p} P_{N_{L}} M_{p}^{*} P_{N_{L}} \geq 0 .
$$


Since $k_{\lambda_{i}}^{L}$ spans $N_{L}$, this holds if and only if

$$
\begin{aligned}
0 & \leq\left[\left(I-P_{N_{L}} M_{p} P_{N_{L}} M_{p}^{*} P_{N_{L}}\right) k_{\lambda_{j}}^{L}, k_{\lambda_{i}}^{L}\right] \\
& =\left[k_{\lambda_{j}}^{L}, k_{\lambda_{i}}^{L}\right]-\left[M_{p}^{*} k_{\lambda_{j}}^{L}, M_{p}^{*} k_{\lambda_{i}}^{L}\right] \\
& =\left[k_{\lambda_{j}}^{L}, k_{\lambda_{i}}^{L}\right]-\left[\overline{w_{j}} k_{\lambda_{j}}^{L}, \overline{w_{i}} k_{\lambda_{i}}^{L}\right] \\
& =\left[\left(1-w_{i} \overline{w_{j}}\right) k^{L}\left(\lambda_{i}, \lambda_{j}\right)\right] .
\end{aligned}
$$

It follows that

$$
\operatorname{dist}\left(p, \mathfrak{J}_{E}\right)=\sup _{L \in \operatorname{CycLat}(\mathfrak{A})}\left\|\left.P_{L} M_{p}^{*}\right|_{N_{L}}\right\| \leq 1 .
$$

The ideal $\mathfrak{J}_{E}$ is weak- $*$-closed, and so the distance is attained at some $g \in \mathfrak{J}$. The multiplier $f:=p-g$ is in the unit ball of $\mathfrak{A}$ and interpolates the given data.

Conversely, suppose that the interpolation property holds. Fix $f \in \mathfrak{A}$ such that $\sup _{L \in \text { CycLat( }(\mathfrak{A})}\left\|\left.P_{L} M_{f}^{*}\right|_{N_{L}}\right\|=1$. By assumption, there is a multiplier $g$ in the unit ball of $\mathfrak{A}$ such that $g\left(\lambda_{i}\right)=f\left(\lambda_{i}\right)$ for each $1 \leq i \leq n$. Hence

$$
\operatorname{dist}\left(f, \mathfrak{J}_{E}\right) \leq\|f-(f-g)\|=\|g\| \leq 1 .
$$

By Lemma 2.8, $\operatorname{dist}\left(M_{f}, \mathfrak{A}\right) \geq\left.\sup _{L \in \operatorname{Lat}(\mathfrak{A})}|| P_{L} M_{f}^{*}\right|_{N_{L}} \|=1$. Therefore the family $\left\{k^{L}: L \in \operatorname{CycLat}(\mathfrak{A})\right\}$ is a Nevanlinna-Pick family of kernels for $\mathfrak{A}$.

We are also interested when the subspaces $M_{L}$ suffice to compute the distance. The same argument shows that these spaces form a Nevanlinna-Pick family for interpolation on the set $E$. This will occur if we can show that cyclic subspaces generated by elements $h$ that do not vanish on $E$ suffice in the distance estimate. This is not always the case, but it does happen in important special cases.

\section{Algebras with property $\mathbb{A}_{1}(1)$}

Let $\mathfrak{A}$ be a dual algebra in $\mathcal{B}(H)$. Given vectors $x$ and $y$ in $H$, let $\left[x y^{*}\right]$ denote the vector functional $A \rightarrow\langle A x, y\rangle$ for $A \in \mathfrak{A}$. Every weak-* continuous functional on $\mathfrak{A}$ is a (generally infinite) linear combination of these vector functionals.

One says that $\mathfrak{A}$ has property $\mathbb{A}_{1}(r)$ if, for each weak-* continuous functional $\varphi$ on $\mathfrak{A}$ with $\|\varphi\|<1$, there are vectors $x$ and $y$ so that $\varphi=\left[x y^{*}\right]$ and $\|x\|\|y\|<r$. Property $\mathbb{A}_{1}(r)$ implies that the weak-* and weak operator topologies of $\mathfrak{A}$ coincide, since the weak operator continuous linearly functionals are precisely the finite linear combinations of vector functionals.

It is well known that for any reproducing kernel Hilbert space, the multiplier algebra $\mathfrak{M}(H)$ is reflexive. This is because $\mathbb{C} k_{\lambda}$ is invariant for $\mathfrak{M}(H)^{*}$ for each kernel function $k_{\lambda}$. Hence, if $T \in \operatorname{Alg}(\operatorname{Lat} \mathfrak{M}(H))$, then $k_{\lambda}$ is an eigenvector for $T^{*}$. It follows that $T$ is a multiplier on $H$ by standard results.

Suppose $\mathfrak{A}$ is a dual subalgebra of $\mathfrak{M}(H)$ that is relatively reflexive; that is, the algebra satisfies

$$
\mathfrak{A}=\operatorname{Alg}(\text { Lat } \mathfrak{A}) \cap \mathfrak{M}(H) .
$$

The reflexivity of the whole multiplier algebra implies that $\mathfrak{A}$ is actually reflexive.

We saw that a multiplier $f$ in $\mathfrak{A}$ defines the multiplication operator $\left.M_{f}\right|_{L}$ on every invariant subspace $L$ of $\mathfrak{A}$. On the other hand, if $f$ is simultaneously a multiplier on each $L$ in Lat $\mathfrak{A}$, then it is clearly in $\mathfrak{M}(H)$ and leaves every $L$ invariant. 
Consequently, $\mathfrak{A}$ is the largest algebra of multipliers for the family of subspaces Lat $(\mathfrak{A})$. It is clear that it suffices to consider cyclic invariant subspaces. So we have

$$
\mathfrak{A}=\bigcap_{L \in \operatorname{Lat}(\mathfrak{A})} \mathfrak{M}(L)=\bigcap_{L \in \operatorname{CycLat}(\mathfrak{A})} \mathfrak{M}(L)
$$

Following the notation of Agler and McCarthy [3], CycLat $\mathfrak{A}$ is called a realizable collection of reproducing kernel Hilbert spaces.

The first part of the following theorem is contained in Hadwin and Nordgren's paper [19, Prop. 2.5(1)] on $\mathbb{A}_{1}(r)$ (which is called $D_{\sigma}(r)$ there). The second part is from Kraus and Larson [23, Theorem 3.3]. We say that a subalgebra $\mathfrak{A}$ of $\mathfrak{B}$ is relatively hyper-reflexive if there is a constant $C$ so that

$$
\operatorname{dist}(B, \mathfrak{A}) \leq C \sup _{L \in \text { Lat } \mathfrak{A}}\left\|P_{L}^{\perp} B P_{L}\right\| \quad \text { for all } \quad B \in \mathfrak{B} .
$$

The optimal value of $C$ is the relative hyper-reflexivity constant. Again, it is clear that cyclic subspaces suffice.

Theorem 3.1 (Hadwin-Nordgren, Kraus-Larson). Suppose $\mathfrak{B}$ is a dual subalgebra of $\mathfrak{M}(H)$ and has property $\mathbb{A}_{1}(r)$. Then every wot-closed unital subalgebra $\mathfrak{A}$ of $\mathfrak{B}$ is reflexive. Moreover, $\mathfrak{A}$ is relatively hyper-reflexive in $\mathfrak{B}$ with distance constant at most $r$.

If $\mathfrak{B}$ has property $\mathbb{A}_{1}(1)$, we obtain an exact distance formula.

Corollary 3.2. Suppose that $\mathfrak{B}$ has property $\mathbb{A}_{1}(1)$, and let $\mathfrak{A}$ be a wOT-closed unital subalgebra. Then

$$
\operatorname{dist}(B, \mathfrak{A})=\sup _{L \in \operatorname{CycLat}(\mathfrak{A})}\left\|P_{L}^{\perp} B P_{L}\right\| \quad \text { for all } \quad B \in \mathfrak{B} .
$$

We can use the same methods to obtain a distance formula to any weak-* closed ideal. We do not have a reference, so we supply a proof. The ideas go back to the seminal work of Sarason [34]. An argument similar to this one is contained in the proof of [17, Theorem 2.1].

Theorem 3.3. Suppose that $\mathfrak{A}$ is a dual algebra on $H$ with property $\mathbb{A}_{1}(1)$, and let $\mathfrak{J}$ be any WOT-closed ideal of $\mathfrak{A}$. Then we obtain

$$
\operatorname{dist}(A, \mathfrak{J})=\sup _{L \in \operatorname{CycLat}(\mathfrak{A})}\left\|\left.P_{L \ominus \mathfrak{J} L} M_{f}^{*}\right|_{L \ominus \mathfrak{J} L}\right\| .
$$

Proof. By Lemma 2.8, dist $(A, \mathfrak{J})$ dominates the right hand side.

Conversely, given $A \in \mathfrak{A}$ and $\varepsilon>0$, pick $\varphi \in \mathfrak{A}_{*}$ such that $\left.\varphi\right|_{\mathfrak{J}}=0,\|\varphi\|<1+\varepsilon$ and $|\varphi(A)|=\operatorname{dist}(A, \mathfrak{J})$. Using property $\mathbb{A}_{1}(1)$, we obtain vectors $x$ and $y$ with $\|x\|=1$ and $\|y\|<1+\varepsilon$ so that $\varphi=\left[x y^{*}\right]$. Set $L=\mathfrak{A}[x]$ in CycLat $\mathfrak{A}$. Since $L$ is invariant, we can and do replace $y$ by $P_{L} y$ without changing $\left[x y^{*}\right]$. Since $\left.\varphi\right|_{\mathfrak{J}}=0$, $y$ is orthogonal to $\mathfrak{J}[x]=\mathfrak{J} L$. Hence $y$ belongs to $L \ominus \mathfrak{J} L$. Therefore

$$
\begin{aligned}
\operatorname{dist}(A, \mathfrak{J}) & =|\langle A x, y\rangle|=\left|\left\langle A P_{L} x, P_{N_{L}} y\right\rangle\right| \\
& =\left|\left\langle P_{L \ominus \mathfrak{J} L} A P_{L} x, y\right\rangle\right|=\left|\left\langle P_{L \ominus \mathfrak{J} L} A P_{L \ominus \mathfrak{J} L} x, y\right\rangle\right| \\
& \leq\left\|P_{L \ominus \mathfrak{J} L} A P_{L \ominus \mathfrak{J} L}\right\|\|x\|\|y\| \\
& <(1+\varepsilon)\left\|\left.P_{L \ominus \mathfrak{J} L} A\right|_{L \ominus \mathfrak{J} L}\right\| .
\end{aligned}
$$

It follows that the right hand side dominates $\operatorname{dist}(A, \mathfrak{J})$. 
We apply this to the context of interpolation in reproducing kernel Hilbert spaces.

Theorem 3.4. Suppose $\mathfrak{A}$ is a dual algebra of multipliers on $H$ with property $\mathbb{A}_{1}(1)$. Let $E$ be a finite subset of $X$ which is separated by $\mathfrak{A}$. Then the following distance formula holds:

$$
\operatorname{dist}\left(f, \mathfrak{J}_{E}\right)=\sup \left\{\left\|\left.P_{N_{L}} M_{f}^{*}\right|_{N_{L}}\right\|: L \in \operatorname{CycLat}(\mathfrak{A})\right\} .
$$

That is, $\left\{k^{L}: L \in\right.$ CycLat $\left.(\mathfrak{A})\right\}$ is a Nevanlinna-Pick family of kernels for $\mathfrak{A}$.

Corollary 3.5. Suppose $\mathfrak{A}$ is a dual algebra of multipliers on a reproducing kernel Hilbert space $H$ on $X$ that has property $\mathbb{A}_{1}(1)$. Let $\left\{\lambda_{1}, \ldots, \lambda_{n}\right\}$ be distinct points in $X$ separated by $\mathfrak{A}$ and let $\left\{w_{1}, \ldots, w_{n}\right\}$ be complex numbers. There is a multiplier $f$ in the unit ball of $\mathfrak{A}$ such that $f\left(\lambda_{i}\right)=w_{i}$ for each $i$ if and only if

$$
\left[\left(1-w_{i} \overline{w_{j}}\right) k^{L}\left(\lambda_{i}, \lambda_{j}\right)\right] \geq 0 \quad \text { for all } L \in \operatorname{CycLat}(\mathfrak{A})
$$

By replacing the property $\mathbb{A}_{1}(1)$ with $\mathbb{A}_{1}(r)$ for $r>1$, one may repeat the proof of the previous theorem and obtain an analogous result where the norm of the interpolant is bounded by $r$.

In applications, is it convenient to use the spaces $M_{L}$ instead of $N_{L}$. In light of Corollary 2.7. this is possible for cyclic subspaces $L=\mathfrak{A}[h]$ provided that $h$ does not vanish on $E$. A refinement of the $\mathbb{A}_{1}(1)$ property can make this possible. The distinction made in this theorem is that the kernel is formed as simply the compressions $P_{L} k_{\lambda}$.

Theorem 3.6. Let $\mathfrak{A}$ be a dual algebra of multipliers on $H$, and let $E=\left\{\lambda_{1}, \ldots, \lambda_{n}\right\}$ be a finite subset of $X$ which is separated by $\mathfrak{A}$. Suppose that $\mathfrak{A}$ has property $\mathbb{A}_{1}(1)$ with the additional stipulation that each weak-* continuous functional $\varphi$ on $\mathfrak{A}$ with $\|\varphi\|<1$ can be factored as $\varphi=\left[x y^{*}\right]$ with $\|x\|\|y\|<1$ such that $x$ does not vanish on $E$. Then there is a multiplier in the unit ball of $\mathfrak{A}$ with $f\left(\lambda_{i}\right)=w_{i}$ for $\lambda_{i} \in E$ if and only if

$$
\left[\left(1-w_{i} \overline{w_{j}}\right)\left\langle P_{L} k_{\lambda_{j}}, k_{\lambda_{i}}\right\rangle\right] \geq 0
$$

for all cyclic subspaces $L=\mathfrak{A}[h]$ where $h$ does not vanish on $E$.

\section{Subalgebras of $H^{\infty}$}

In this section, we discuss the algebra $H^{\infty}$ and its subalgebras. In particular, we show how to recover results of Davidson, Paulsen, Raghupathi and Singh [14] and Raghupathi [31. We also show how one can formulate a Nevanlinna-Pick theorem for $H^{\infty}$ on Bergman space. While this is only of theoretical interest, since interpolation is better done on the Hardy space, this has been an open question for some time.

Hardy space. Let $H=H^{2}$ be Hardy space, so that $\mathfrak{M}(H)=H^{\infty}$. It is well known that this algebra has property $\mathbb{A}_{1}(1)$ [8, Theorem 3.7].

Moreover, any weak-* continuous functional $\varphi$ on $H^{\infty}$ can be factored exactly as $\varphi=\left[x y^{*}\right]$ with $\|x\|\|y\|=\|\varphi\|$. Now if the inner-outer factorization of $x$ is $x=\omega h$, with $h$ outer and $\omega$ inner, then $\varphi=\left[h k^{*}\right]$ where $k=M_{\omega}^{*} y$. Thus the factorization may be chosen so that $h$ is outer, and in particular, does not vanish on $\mathbb{D}$.

Consider the classical Nevanlinna-Pick problem for a finite subset $E$ of $\mathbb{D}$. Following Sarason's approach [34], let $B_{E}$ denoting the finite Blaschke product with 
simple zeros at $E$. Then $\mathfrak{J}_{E}=B_{E} H^{\infty}$. Beurling's invariant subspace theorem for the unilateral shift shows that if $L \in \operatorname{Lat}\left(H^{\infty}\right)$, then there is some inner function $\omega$ so that $L=\omega H^{2}$. In particular, every invariant subspace is cyclic. Observe that

$$
N_{\omega}:=\omega H^{2} \ominus \omega B_{E} H^{2}=\omega\left(H^{2} \ominus B_{E} H^{2}\right)=\omega M(E) .
$$

Here $M(E)=\operatorname{span}\left\{k_{\lambda}: \lambda \in E\right\}=H^{2} \ominus B_{E} H^{2}$. However $M_{\omega}$ is an isometry in $\mathfrak{M}\left(H^{2}\right)$, and hence commutes with $\mathfrak{M}\left(H^{2}\right)$. So it provides a unitary equivalence between the compression of $\mathfrak{M}\left(H^{2}\right)$ to $N_{\omega}$ and to $M(E)$.

Therefore, for $f \in H^{\infty}$, Theorem 3.4 gives

$$
\operatorname{dist}\left(f, B_{E} H^{\infty}\right)=\sup \left\{\left\|\left.P_{N_{\omega}} M_{f}^{*}\right|_{N_{\omega}}\right\|: \omega \text { inner }\right\}=\left\|\left.M_{f}^{*}\right|_{M(E)}\right\| .
$$

The classical Nevanlinna-Pick interpolation theorem now follows as in Corollary 3.5 as Sarason showed.

Perhaps more importantly, Theorem 3.4 provides an interpolation theorem for any weak-*-closed subalgebra of $H^{\infty}$. These constrained Nevanlinna-Pick theorems fashion suitable generalizations of the results seen in [14] and [31].

In fact, since any weak-* linear functional $\varphi$ on $\mathfrak{A}$ extends to a functional on $H^{\infty}$ with arbitrarily small increase in norm, it can be factored as $\varphi=\left[h k^{*}\right]$ where $h$ is outer and $\|h\|\|k\|<\|\varphi\|+\varepsilon$. Therefore the more refined Theorem 3.6] applies. We obtain:

Theorem 4.1. Let $\mathfrak{A}$ be a weak-*-closed unital subalgebra of $H^{\infty}$. Let $E=$ $\left\{\lambda_{1}, \ldots, \lambda_{n}\right\}$ be a finite subset of $\mathbb{D}$ which is separated by $\mathfrak{A}$. Then there is a multiplier in the unit ball of $\mathfrak{A}$ with $f\left(\lambda_{i}\right)=w_{i}$ for $1 \leq i \leq n$ if and only if

$$
\left[\left(1-w_{i} \overline{w_{j}}\right)\left\langle P_{L} k_{\lambda_{i}}, k_{\lambda_{j}}\right\rangle\right] \geq 0
$$

for all cyclic subspaces $L=\mathfrak{A}[h]$ where $h$ is outer.

In [14], the algebra $H_{1}^{\infty}=\left\{f \in H^{\infty}: f^{\prime}(0)=0\right\}$ is studied. Beurling's Theorem was used to show that there is a simple parameterization of the cyclic invariant subspaces $H_{1}^{\infty}[h]$ for $h$ outer, namely

$$
H_{\alpha, \beta}^{2}:=\operatorname{span}\left\{\alpha+\beta z, z^{2} H^{2}\right\} \text { for all }(\alpha, \beta) \text { with }|\alpha|^{2}+|\beta|^{2}=1 .
$$

It was shown that these provide a Nevanlinna-Pick family for $H_{1}^{\infty}$, and the consequent interpolation theorem. Raghupathi [31] carries out this program for the class of algebras $H_{B}^{\infty}=\mathbb{C} 1+B H^{\infty}$ where $B$ is a finite Blaschke product.

In [32], Raghupathi shows that Abrahamse's interpolation result for multiply connected domains [1] is equivalent to the interpolation problem for certain weak* subalgebras of $H^{\infty}$. Consequently, our distance formula includes Abrahamse's theorem as a special case.

Singly-generated multiplier algebras. Suppose that $T$ is an absolutely continuous contraction on $H$, and let $\mathfrak{A}_{T}$ be the unital, weak-*-closed algebra generated by $T$. The Sz.Nagy dilation theory provides a weak-* continuous, contractive homomorphism $\Phi: H^{\infty} \rightarrow \mathfrak{A}_{T}$ given by $\Phi(f)=\left.P_{H} f(U)\right|_{H}$, where $U$ is the minimal unitary dilation of $T$. If $\Phi$ is isometric (i.e. $\|\Phi(f)\|=\|f\|_{\infty}$ for every $f \in H^{\infty}$ ), then $\Phi$ is also a weak-* homeomorphism. In addition, $\Phi$ being isometric ensures that the preduals $\left(\mathfrak{A}_{T}\right)_{*}$ and $L_{1} / H_{0}^{1}$ are isometrically isomorphic. In this case, we say that $T$ has an isometric functional calculus. See [9] for relevant details. The following deep result of Bercovici [11] will be used. 
Theorem 4.2 (Bercovici). Suppose $T$ is an absolutely continuous contraction on $H$ and that $T$ has an isometric functional calculus. Then $\mathfrak{A}_{T}$ has property $\mathbb{A}_{1}(1)$.

We will use Theorem 4.2 to show that a wide class of reproducing kernel Hilbert spaces admit Nevanlinna-Pick families of kernels for arbitrary dual subalgebras of $H^{\infty}$.

Example 4.3. Let $\mu$ be a finite Borel measure on $\mathbb{D}$, and let $P^{2}(\mu)$ denote the closure of the polynomials in $L^{2}(\mu)$. A bounded point evaluation for $P^{2}(\mu)$ is a point $\lambda$ for which there exists a constant $M>0$ with $|p(\lambda)| \leq M\|p\|_{P^{2}(\mu)}$ for every polynomial $p$. A point $\lambda$ is said to be an analytic bounded point evaluation for $P^{2}(\mu)$ if $\lambda$ is in the interior of the set of bounded point evaluatons, and that the map $z \rightarrow f(z)$ is analytic on a neighborhood of $\lambda$ for every $f \in P^{2}(\mu)$.

It follows that if $\lambda$ is a bounded point evaluation, then there is a kernel function $k_{\lambda}$ in $P^{2}(\mu)$ so that $p(\lambda)=\left\langle p, k_{\lambda}\right\rangle$. For an arbitrary $f \in P^{2}(\mu)$, if we set $f(\lambda)=$ $\left\langle f, k_{\lambda}\right\rangle$, then these values will agree with $f$ a.e. with respect to $\mu$. For both the Hardy space and Bergman space, the set of analytic bounded point evaluation is all of $\mathbb{D}$. A theorem of Thomson shows that either $P^{2}(\mu)=L^{2}(\mu)$ or $P^{2}(\mu)$ has analytic bounded point evaluations [35].

Let $m$ be Lebesque area measure on the disk. For $s>0$, define a weighted area measure on $\mathbb{D}$ by $d \mu_{s}(z)=(1-|z|)^{s-1} d m(z)$. The monomials $z^{n}$ form an orthogonal basis for $P^{2}(\mu)$. This includes the Bergman space for $s=1$. For these spaces, every point in $\mathbb{D}$ is an analytic point evaluation.

The following result appears as Theorem 4.6 in [3].

Theorem 4.4. Let $\mu$ be a measure on $\mathbb{D}$ such that the set of analytic bounded point evaluations of $P^{2}(\mu)$ contains all of $\mathbb{D}$. Then $\mathfrak{M}\left(P^{2}(\mu)\right)$ is isometrically isomorphic and weak-* homeomorphic to $H^{\infty}$.

Corollary 3.5 yields the following interpolation result for these spaces.

Theorem 4.5. Let $\mu$ be a measure on $\mathbb{D}$ such that the set of analytic bounded point evaluations of $P^{2}(\mu)$ contains all of $\mathbb{D}$. Suppose $\mathfrak{A}$ is a dual subalgebra of $\mathfrak{M}\left(P^{2}(\mu)\right)$. Then $\mathfrak{A}$ has a Nevanlinna-Pick family of kernels.

Example 4.6. In particular, Theoorem 4.5 provides a Nevanlinna-Pick condition for Bergman space $A^{2}:=A^{2}(\mathbb{D})$, whose reproducing kernel kernel $k_{\lambda}^{B}=(1-\bar{\lambda} z)^{-2}$ is not an NP kernel. In fact, the Bergman kernel fails to even have the twopoint Pick property. See [3, Example 5.17] for details. The multiplier algebra of Bergman space has property $\mathbb{A}_{1}(1)$ as a consequence of much stronger properties (see Section 7), but the subspace lattice of the Bergman shift is immense.

\section{Complete NP KeRnels}

A reproducing kernel is a complete Nevanlinna-Pick kernel if matrix interpolation is determined by the positivity of the Pick matrix for the data. That is, if $H$ is a Hilbert space with reproducing kernel $k$ on a set $X, E=\left\{\lambda_{1}, \ldots, \lambda_{n}\right\}$ is a finite subset of $X$, and $W_{1}, \ldots, W_{n}$ are $r \times r$ matrices, then a necessary condition for there to be an element $F \in \mathcal{M}_{r}(\mathfrak{M}(H))$ with $F\left(\lambda_{i}\right)=W_{i}$ and $\|F\| \leq 1$ is the positivity of the matrix

$$
\left[\left(I_{r}-W_{i} W_{j}^{*}\right) k\left(\lambda_{i}, \lambda_{j}\right)\right]
$$


We say that $k$ is a complete NP kernel if this is also sufficient.

The first author and Pitts [17] showed that symmetric Fock space, now called the Drury-Arveson space $H_{d}^{2}$, on the complex ball $\mathbb{B}_{d}$ of $\mathbb{C}^{d}$ (including $d=\infty$ ) is a complete NP space with kernel

$$
k(w, z)=\frac{1}{1-\langle w, z\rangle} .
$$

The complete NP kernels were classified by McCullough[24, 25] and Quiggin [29, 30] building on work by Agler (unpublished). Another proof was provided by Agler and McCarthy [2], who noticed the universality of the Drury-Arveson kernel.

Theorem 5.1 (McCullough, Quiggin, Agler-McCarthy). Let $k$ be an irreducible kernel on $X$. Then $k$ is a complete Nevanlinna-Pick kernel if and only if for some cardinal $d$, there is an injection $b: X \rightarrow \mathbb{B}_{d}$ and a nowhere-vanishing function $\delta: X \rightarrow \mathbb{C}$ such that

$$
k(x, y)=\frac{\overline{\delta(x)} \delta(y)}{1-\langle b(x), b(y)\rangle} .
$$

In this case, the map $k_{x} \rightarrow \frac{\delta(x)}{1-\langle z, b(x)\rangle}$ extends to an isometry of $H$ into $H_{d}^{2}$.

Since the span of kernel functions is always a co-invariant subspace for the space of multipliers, the complete NP kernel spaces are seen to correspond to certain co-invariant subspaces of Drury-Arveson space, i.e. $\operatorname{span}\left\{k_{z}: z \in b(X)\right\}$. The first author and Pitts [17] show that the multiplier algebras of these spaces are all complete quotients of the non-commutative analytic Toeplitz algebra, $\mathfrak{L}_{d}$, generated by the left regular representation of the free semigroup $\mathbb{F}_{d}^{+}$on the full Fock space $\ell^{2}\left(\mathbb{F}_{d}^{+}\right)$. It follows immediately that they are complete quotients of $\mathfrak{M}\left(H_{d}^{2}\right)$. See also Arias and Popescu [6].

We will show that all such quotients of $\mathfrak{M}\left(H_{d}^{2}\right)$ have property $\mathbb{A}_{1}(1)$. The algebra $\mathfrak{L}_{d}$ actually has property $\mathbb{A}_{\aleph_{0}}[\mathbf{1 5}]$ and even property $X_{0,1}[\mathbf{1 2}]$. But these stronger properties do not extend to $\mathfrak{M}\left(H_{d}^{2}\right)$.

More specifically, if $\mathfrak{J}$ is a wOT-closed ideal of $\mathfrak{L}_{d}$ with range $M=\overline{\mathfrak{J} \ell^{2}\left(\mathbb{F}_{d}^{+}\right)}$, then [17] shows that $\mathfrak{L}_{d} / \mathfrak{J}$ is completely isometrically isomorphic to the compression of $\mathfrak{L}_{d}$ to $M^{\perp}$. Conversely, if $M$ is an invariant subspace of both $\mathfrak{L}_{d}$ and its commutant, the right regular representation algebras $\mathfrak{R}_{d}$, then $\mathfrak{J}=\left\{A \in \mathfrak{L}_{d}: \operatorname{Ran} A \subset M\right\}$ is a WOT-closed ideal with range $M$. In particular, if $\mathfrak{C}$ is the commutator ideal, it is shown that $\mathfrak{M}\left(H_{d}^{2}\right) \simeq \mathfrak{L}_{d} / \mathfrak{C}$. Moreover the compression of both $\mathfrak{L}_{d}$ and $\mathfrak{R}_{d}$ to $H_{d}^{2}$ agree with $\mathfrak{M}\left(H_{d}^{2}\right)$. So if $N$ is a coinvariant subspace of $H_{d}^{2}$, then $M=\ell^{2}\left(\mathbb{F}_{d}^{+}\right) \ominus N$ is invariant for both $\mathfrak{L}_{d}$ and $\mathfrak{R}_{d}$ and the theory applies.

The following result is due to Arias and Popescu [6, Prop.1.2]. We provide a proof for completeness.

Theorem 5.2 (Arias-Popescu). Let $\mathfrak{J}$ be any wot-closed ideal of $\mathfrak{L}_{d}$ and let $M=$ $\overline{\mathfrak{J} \ell^{2}\left(\mathbb{F}_{d}^{+}\right)}$. Then $\mathfrak{A}=\left.P_{M}^{\perp} \mathfrak{L}_{d}\right|_{M^{\perp}}$ has property $\mathbb{A}_{1}(1)$.

Proof. Let $q: \mathfrak{L}_{d} \rightarrow \mathfrak{L}_{d} / \mathfrak{J} \simeq \mathfrak{A}$ denote the canonical quotient map. Suppose $\varphi$ is a weak-* functional on $\mathfrak{A}$ with $\|\varphi\|<1$. Then $\varphi \circ q$ is a weak-* continuous functional on $\left(\mathfrak{L}_{d}\right)_{*}$ of norm less than 1 . Hence there are vectors $x$ and $y$ in $\ell^{2}\left(\mathbb{F}_{d}^{+}\right)$with $\varphi \circ q=\left[x y^{*}\right]$ and $\|x\|\|y\|<1$. Form the cyclic subspace $L=\mathfrak{L}_{d}[x]$. By [5, 15] there is an isometry $R \in \mathfrak{R}_{d}$ such that $\mathfrak{L}_{d}[x]=R \ell^{2}\left(\mathbb{F}_{d}^{+}\right)$. Let $u$ be the vector such 
that $x=R u$ and set $v=R^{*} y$. A direct calculation shows that $\left[x y^{*}\right]=\left[u v^{*}\right]$. Observe that $\mathfrak{L}_{d}[u]=\ell^{2}\left(\mathbb{F}_{d}^{+}\right)$.

Obviously $\left[u v^{*}\right]$ annihilates $\mathfrak{J}$, and it follows that $v$ is orthogonal to

$$
\overline{\mathfrak{J} u}=\overline{\mathfrak{J} \mathfrak{L}_{d} u}=\overline{\mathfrak{J} \ell^{2}\left(\mathbb{F}_{d}^{+}\right)}=M .
$$

Thus $v \in M^{\perp}$. Now, for $A \in \mathfrak{A}$, pick $B \in \mathfrak{L}_{n}$ with $A=q(B)=\left.P_{M}^{\perp} B\right|_{M} ^{\perp}$. Then since $M^{\perp}$ is coinvariant for $\mathfrak{L}_{d}$,

$$
\begin{aligned}
\varphi(A) & =\varphi \circ q(B)=\langle B u, v\rangle \\
& =\left\langle B u, P_{M}^{\perp} v\right\rangle=\left\langle P_{M}^{\perp} B P_{M}^{\perp} u, v\right\rangle \\
& =\left\langle A\left(P_{M}^{\perp} u\right), v\right\rangle .
\end{aligned}
$$

Hence $\varphi=\left[\left(P_{M}^{\perp} u\right) v^{*}\right]$. Therefore $\mathfrak{A}$ has property $\mathbb{A}_{1}(1)$.

The remarks preceding this theorem show that the multiplier algebra of every complete NP kernel arise in this way. We further refine this to observe that the vector $x$ in the factorization may be chosen so that it does not vanish.

Corollary 5.3. The multiplier algebra $\mathcal{M}(H)$ of every complete $N P$ kernel has property $\mathbb{A}_{1}(1)$. In particular, $\mathfrak{M}\left(H_{d}^{2}\right)$ has property $\mathbb{A}_{1}(1)$. Moreover, each $\varphi \in$ $\mathfrak{M}(H)_{*}$ with $\|\varphi\|<1$ can be represented as $\varphi=\left[x y^{*}\right]$ such that $x$ does not vanish on $X$ and $\|x\|\|y\|<1$.

Proof. We may assume that $\mathcal{M}(H)$ is the compression of $\mathfrak{L}_{d}$ to the subspace $M=\operatorname{span}\left\{k_{\lambda}: \lambda \in X \subset \mathbb{B}_{n}\right\}$. It remains only to verify that in the proof of Theorem 5.2. the function $P_{M}^{\perp} u$ does not vanish. Since $u$ is a cyclic vector for $\mathfrak{L}_{d}$, it is not orthogonal to any $k_{\lambda}$ because $\mathbb{C} k_{\lambda}$ is coinvariant. Therefore for any $\lambda \in X$,

$$
0 \neq\left\langle u, k_{\lambda}\right\rangle=\left\langle u, P_{M}^{\perp} k_{\lambda}\right\rangle=\left\langle P_{M}^{\perp} u, k_{\lambda}\right\rangle=\delta(\lambda)^{-1}\left(P_{M}^{\perp} u\right)(\lambda),
$$

where $\delta$ is the scaling function of the embedding in Theorem 5.1 .

The proof actually shows that it suffices to use vectors $h$ which are cyclic for $\mathfrak{M}(H)$. In the case of Drury-Arveson space, like for Hardy space, these vectors are called outer.

Remark 5.4. The algebra $\mathfrak{M}\left(H_{d}^{2}\right)$ does not have property $\mathbb{A}_{\aleph_{0}}$. The reason is that algebras with this property have non-trivial invariant subspaces which are orthogonal [10]. But any two non-trivial invariant subspaces of $\mathfrak{M}\left(H_{d}^{2}\right)$ have nontrivial intersection. Indeed, if $M \in$ Lat $\mathfrak{M}\left(H_{d}^{2}\right)$, then $N=M+\left(H_{d}^{2}\right)^{\perp}$ is invariant for $\mathfrak{L}_{d}$. By [5, 15], this space is the direct sum of cyclic invariant subspaces $N_{i}=R_{i} \ell^{2}\left(\mathbb{F}_{d}^{+}\right)$for isometries $R_{i} \in \mathfrak{R}_{d}$. The compression $\left.P_{H_{d}^{2}} R_{i}\right|_{H_{d}^{2}}$ is a multiplier $M_{f_{i}}$ in $\mathfrak{M}\left(H_{d}^{2}\right)$. Thus

$$
\begin{aligned}
M & =P_{H_{d}^{2}} N=\sum_{i} P_{H_{d}^{2}} R_{i} \ell^{2}\left(\mathbb{F}_{d}^{+}\right) \\
& =\sum_{i} P_{H_{d}^{2}} R_{i} P_{H_{d}^{2}} \ell^{2}\left(\mathbb{F}_{d}^{+}\right)=\sum_{i} M_{f_{i}} H_{d}^{2} .
\end{aligned}
$$

In particular, every invariant subspace $M$ contains the range of a non-zero multiplier $M_{f}$. Hence given two invariant subspaces $M$ and $N$ in $H_{d}^{2}$, we can find non-zero multipliers $f$ and $g$ with $\operatorname{Ran} M_{f} \subset M$ and $\operatorname{Ran} M_{g} \subset N$. So $M \cap N$ contains $\operatorname{Ran} M_{f g}$. 
Based on heuristic calculations, we expect that $\mathfrak{M}\left(H_{d}^{2}\right)$ does not have property $\mathbb{A}_{2}(r)$ for any $r \geq 1$, and likely not $\mathbb{A}_{2}$.

We are now in a position to apply Theorem 3.6.

Theorem 5.5. Suppose $k$ is an irreducible, complete Nevanlinna-Pick kernel on $X$. Then any dual subalgebra $\mathfrak{A}$ of multipliers of $H$ admits a Nevanlinna-Pick family of kernels. More specifically, if $E=\left\{\lambda_{1}, \ldots, \lambda_{n}\right\}$ is a finite subset of $X$ which is separated by $\mathfrak{A}$ and $w_{1}, \ldots, w_{n}$ are scalars, then there is a multiplier $f$ in the unit ball of $\mathfrak{A}$ with $f\left(\lambda_{i}\right)=w_{i}$ for $1 \leq i \leq n$ if and only if

$$
\left[\left(1-w_{i} \overline{w_{j}}\right)\left\langle P_{L} k_{\lambda_{j}}, k_{\lambda_{i}}\right\rangle\right] \geq 0
$$

for all cyclic invariant subspaces $L=\mathfrak{A}[h]$ of $\mathfrak{A}$ (and it suffices to use $h$ which do not vanish on $E)$.

\section{Finite KeRnels}

In this section, we present numerical evidence that there is a finite dimensional multiplier algebra $\mathfrak{A}$ with the property that CycLat( $\mathfrak{A})$ is not an NP family for $\mathfrak{A}$. In particular, this algebra does not have $\mathbb{A}_{1}(1)$. It does, however, have property $\mathbb{A}_{1}(r)$ for some $r>1$.

Suppose $X=\left\{\lambda_{1}, \ldots, \lambda_{N}\right\}$ is a finite set and $k: X \times X \rightarrow \mathbb{C}$ is an irreducible kernel. Let $y_{1}, \ldots, y_{N}$ be vectors in $\mathbb{C}^{N}$ such that $k\left(\lambda_{i}, \lambda_{j}\right)=\left\langle y_{j}, y_{i}\right\rangle$, and let $\left\{x_{1}, \ldots, x_{N}\right\}$ be a dual basis for the $y_{i}$. The space $H=\mathbb{C}^{N}$ may be regarded as a reproducing kernel Hilbert space over $X$, with reproducing kernel at $\lambda_{i}$ given by $y_{i}$. The multiplier algebra $\mathfrak{M}(H)$ is an $N$-idempotent operator algebra spanned by the rank one idempotents $p_{i}=x_{i} y_{i}^{*}$.

If $\left\{e_{i}\right\}$ is the canonical orthonormal basis for $\mathbb{C}^{N}$, then one readily sees that $\mathfrak{M}(H)$ is similar to the diagonal algebra $\mathfrak{D}_{N}$ via the similarity $S$ defined by $S e_{i}=x_{i}$. Since $\mathfrak{D}_{N}$ evidently has property $\mathbb{A}_{1}(1)$, it follows from elementary results on dual algebras that $\mathfrak{M}(H)$ has $\mathbb{A}_{1}(r)$ for some $r \geq 1$.

If $k$ is irreducible and a complete NP kernel, then Corollary 5.3 shows that $\mathfrak{M}(H)$ has $\mathbb{A}_{1}(1)$. However, there are many kernels $k$ that cannot be embedded in DruryArveson space in this way. We expect that many of these algebras fail to have $\mathbb{A}_{1}(1)$ and that the distance formula fails in such cases.

Since $\mathfrak{A}$ is similar to the diagonal algebra $\mathfrak{D}_{N}$, the invariant subspaces are spanned by some subset of $\left\{x_{1}, \ldots, x_{N}\right\}$. Denote them by $L_{\sigma}=\operatorname{span}\left\{x_{i}: i \in \sigma\right\}$. For $E \subset\{1, \ldots, N\}$, the ideal $\mathfrak{J}=\mathfrak{J}_{E}=\operatorname{span}\left\{p_{i}: i \notin E\right\}$. Then $\mathfrak{J} L_{\sigma}=L_{\sigma \backslash E}$, and $N_{\sigma}:=N_{L_{\sigma}}=L_{\sigma} \ominus L_{\sigma \backslash E}$. The distance formula is obtained as the maximum of compressions to these subspaces - so we need only consider the maximal ones. These arise from $\sigma \supset E$.

For trivial reasons, the distance formula is always satisfied when $N=2$ and $N=3$. There is strong numerical evidence to suggest that the formula does hold for $N=4$, though we do not have a proof. In the following 5 -dimensional example, Wolfram Mathematica 7 was used to find a similarity $S$ such that the distance formula fails. 
Example 6.1. Define the similarity

$$
S=\left[\begin{array}{rrrrr}
3 & 1 & 1 & 0 & -1 \\
0 & 1 & -2 & -1 & 0 \\
-1 & 0 & -1 & 1 & -1 \\
-1 & 1 & 2 & 1 & -1 \\
1 & 1 & 3 & 1 & -2
\end{array}\right]
$$

Let $p_{i}=x_{i} y_{i}^{*}$ for $1 \leq i \leq 5$ be the idempotents which span the algebra $\mathfrak{A}:=\mathfrak{M}(H)$. Let $E=\{1,2,3\}$, and form $\mathfrak{J}=\mathfrak{J}_{E}=\operatorname{span}\left\{p_{4}, p_{5}\right\}$.

Consider the element $A=-2 p_{1}-3 p_{2}+7 p_{3}$. We are interested in comparing $\max _{\sigma}\left\|P_{N_{\sigma}} A P_{N_{\sigma}}\right\|$ with $\operatorname{dist}(A, \mathfrak{J})$. As noted above, it suffices to use maximal $N_{\sigma}$ 's formed by the cyclic subspaces that do not vanish on $E$, namely

$$
\begin{aligned}
N_{\{123\}} & =\operatorname{span}\left\{x_{1}, x_{2}, x_{3}\right\}, \\
N_{\{1234\}} & =\operatorname{span}\left\{x_{1}, x_{2}, x_{3}, x_{4}\right\} \ominus \mathbb{C} x_{4}, \\
N_{\{1235\}} & =\operatorname{span}\left\{x_{1}, x_{2}, x_{3}, x_{5}\right\} \ominus \mathbb{C} x_{5}, \text { and } \\
N_{\{12345\}} & =\operatorname{span}\left\{x_{4}, x_{5}\right\}^{\perp}=\operatorname{span}\left\{y_{1}, y_{2}, y_{3}\right\} .
\end{aligned}
$$

For notational convenience, set $P_{\sigma}:=P_{N_{\sigma}}$. The values of $\left\|P_{\sigma} A P_{\sigma}\right\|$ were computed and rounded to four decimal places:

$$
\begin{aligned}
\left\|P_{123} A P_{123}\right\| & =9.0096, \\
\left\|P_{1234} A P_{1234}\right\| & =10.1306, \\
\left\|P_{1235} A P_{1235}\right\| & =7.4595, \\
\left\|P_{12345} A P_{12345}\right\| & =10.6632 .
\end{aligned}
$$

By minimizing a function of two variables, the following distance estimate was obtained

$$
\operatorname{dist}(A, \mathfrak{J}) \approx 11.9346 \text {. }
$$

Similar results appeared for many different elements of $\mathfrak{A}$, which indicate that CycLat $(\mathfrak{A})$ is not an NP family for $\mathfrak{A}$. Consequently, it must also fail to have $\mathbb{A}_{1}(1)$. We currently have no example of a dual algebra of multipliers on any $H$ that fails to have $\mathbb{A}_{1}(r)$ for every $r \geq 1$, or even fails to have $\mathbb{A}_{1}$.

\section{Matrix-VAlued Interpolation}

In this section, we will discuss matrix-valued Nevanlinna-Pick interpolation problems. The classical theorem for matrices says that given $z_{1}, \ldots, z_{n}$ in the disk, and $r \times r$ matrices $W_{1}, \ldots, W_{n}$, there is a function $F$ in the the unit ball of $\mathcal{M}_{r}\left(H^{\infty}\right)$ such that $F\left(z_{i}\right)=W_{i}$ if and only if the Pick matrix

$$
\left[\frac{I_{r}-W_{i} W_{j}^{*}}{1-z_{i} \overline{z_{j}}}\right]_{r \times r}
$$

is positive semidefinite.

One can define a linear map $R$ on $M(E) \otimes \mathbb{C}^{r}$ by setting

$$
R\left(k_{\lambda_{i}}^{s} \otimes u\right)=k_{\lambda_{i}}^{s} \otimes W_{i}^{*} u \quad \text { for } \quad 1 \leq i \leq n \text { and } u \in \mathbb{C}^{r} .
$$


Note that if $F$ is an arbitrary interpolant, then $R=\left.M_{F}^{*}\right|_{M(E) \otimes \mathbb{C}^{r}}$. Now $\|R\| \leq 1$ is equivalent to $I-R^{*} R \geq 0$, which is equivalent to the positivity condition above. Hence, this provides the complete distance formula:

$$
\operatorname{dist}\left(F, \mathcal{M}_{r}(\mathfrak{J})\right)=\left\|\left.M_{F}^{*}\right|_{M(E) \otimes \mathbb{C}^{r}}\right\| .
$$

The same holds (by definition) for all complete NP kernels when the factor $\frac{1}{1-z_{i} \overline{z_{j}}}$ is replaced by $k\left(\lambda_{i}, \lambda_{j}\right)$. The multiplier algebra of all complete NP kernels therefore satisfy the analogous distance formula.

Our goal is to generalize the results of Section 2 to a matrix-valued setting by imposing stronger conditions on our algebras of multipliers. Let $(H, k)$ be a reproducing kernel Hilbert space over $X$, and let $r \geq 1$. We will consider the algebra $\mathcal{M}_{r}(\mathfrak{M}(H))$ of $r \times r$ matrices of multipliers acting on the vector-valued space $H^{(r)}=H \otimes \mathbb{C}^{r}$.

For any non-zero vector $u \in \mathbb{C}^{r}$, the functions $k_{\lambda} \otimes u$ act as vector-valued kernel functions. We can therefore consider $\mathcal{M}_{r}(\mathfrak{M}(H))$ as functions on $X$ with values in $\mathcal{M}_{r}$. They act as multipliers of $H^{(r)}$, and inherit a norm as elements of $\mathcal{M}_{r}(\mathcal{B}(H)) \simeq \mathcal{B}\left(H^{(r)}\right)$.

It is readily verified that for any multiplier $F$ and any $\lambda \in X$, we have

$$
M_{F}^{*}\left(k_{\lambda} \otimes u\right)=k_{\lambda} \otimes F(\lambda)^{*} u \quad \text { for } \quad \lambda \in X \text { and } u \in \mathbb{C}^{r} .
$$

Conversely, any bounded operator on $H^{(r)}$ that satisfies these relations is a multiplier of $H^{(r)}$. The algebra $\mathcal{M}_{r}(\mathfrak{M}(H))$ is a unital, weak-*-closed subalgebra of $\mathcal{M}_{r}(\mathcal{B}(H))$, and thus is a dual algebra. Consequently, we may apply the same heuristic as Section 2 when trying to compute distances.

Any dual subalgebra $\mathfrak{A}$ of $\mathfrak{M}(H)$ determines the dual subalgebra $\mathcal{M}_{r}(\mathfrak{A})$ of $\mathcal{M}_{r}(\mathfrak{M}(H))$. Suppose that $E=\left\{\lambda_{i}: 1 \leq i \leq n\right\}$ is a finite subset of $X$ separated by $\mathfrak{A}$. Let $\mathfrak{J}_{E}$ be the ideal of functions in $\mathfrak{A}$ vanishing on $E$. For $F \in \mathcal{M}_{r}(\mathfrak{A})$, any subspace of the form $L^{(r)}$ for $L \in \operatorname{Lat}(\mathfrak{A})$ is invariant for $\mathcal{M}_{r}(\mathfrak{A})$. Conversely, any invariant subspace of $\mathcal{M}_{r}(\mathfrak{A})$ takes this form.

The subspace $L^{(r)}$ is cyclic if and only if $L$ is $r$-cyclic because if $x_{1}, \ldots, x_{r}$ is a cyclic set, then $x=\left(x_{1}, \ldots, x_{r}\right)$ is a cyclic vector for $L^{(r)}$ and vice versa. So in general we cannot deal only with cyclic invariant subspaces of the algebra $\mathfrak{A}$. We will have to deal with some multiplicity of the kernels on these spaces. This can be handled as in the discussion in Remark 2.9 .

We can apply Lemma 2.8 to $\mathcal{M}_{r}(\mathfrak{A})$ and the ideal $\mathcal{M}_{r}\left(\mathfrak{J}_{E}\right)$. For any $F \in \mathcal{M}_{r}(\mathfrak{A})$, we have

$$
\begin{aligned}
\operatorname{dist}\left(F, \mathcal{M}_{r}\left(\mathfrak{J}_{E}\right)\right) & \geq \sup _{L \in \operatorname{Lat}(\mathfrak{A})}\left\|\left(P_{N_{L}} \otimes I_{r}\right) M_{F}\left(P_{N_{L}} \otimes I_{r}\right)\right\| \\
& \geq \sup _{L \in \operatorname{Lat}(\mathfrak{A})}\left\|\left(P_{M_{L}} \otimes I_{r}\right) M_{f}\left(P_{M_{L}} \otimes I_{r}\right)\right\| .
\end{aligned}
$$

Definition 7.1. If equality holds for this distance formula for every finite subset $E$ which is separated by $\mathfrak{A}$, i.e. for any $F \in \mathcal{M}_{r}(\mathfrak{A})$, and $N_{L}=L \ominus \mathfrak{J}_{E} L$ for $L \in$ Lat $\mathfrak{A}$,

$$
\operatorname{dist}\left(F, \mathcal{M}_{r}\left(\mathfrak{J}_{E}\right)\right)=\sup _{L \in \operatorname{Lat}(\mathfrak{A})}\left\|\left(P_{N_{L}} \otimes I_{r}\right) M_{F}\left(P_{N_{L}} \otimes I_{r}\right)\right\|
$$

then we say that Lat $\mathfrak{A}$ is an $r \times r$ Nevanlinna-Pick family for $\mathfrak{A}$. If this holds for all $r \geq 1$, then we say that Lat $\mathfrak{A}$ is a complete Nevanlinna-Pick family for $\mathfrak{A}$. 
Generally, property $\mathbb{A}_{1}(1)$ is not inherited by matrix algebras. Conway and Ptak 13. show that any absolutely continuous contraction in class $C_{00}$ with an isometric functional calculus has $X_{0,1}$. This includes the Bergman shift $B$, and consequently the multiplier algebra of Bergman space. The property $X_{0,1}$ implies that $\mathcal{M}_{r}(\mathfrak{A})$ has $\mathbb{A}_{1}(1)$ for every $r \geq 1$.

On the other hand, it can be the case that some finite ampliation of the algebra will have $\mathbb{A}_{1}(1)$. Given a dual algebra $\mathfrak{A}$ on a Hilbert space $H$, the $k$-th ampliation $\mathfrak{A}^{(k)}$ is an isometric representation of $\mathfrak{A}$ on $H^{(k)}$, the direct sum of $k$ copies of $H$, with elements $A^{(k)}=A \oplus \cdots \oplus A$, the direct sum of $k$ copies of $A$. The preduals $\mathfrak{A}_{*}$ and $\mathfrak{A}_{*}^{(k)}$ are isometrically isomorphic. However, a rank $k$ functional on $\mathfrak{A}$ converts to a rank one functional on $\mathfrak{A}^{(k)}$ since

$$
\sum_{i=1}^{k}\left\langle A x_{i}, y_{i}\right\rangle=\left\langle T^{(k)} \mathbf{x}, \mathbf{y}\right\rangle
$$

where $\mathbf{x}=\left(x_{1}, \ldots, x_{k}\right)$ and $\mathbf{y}=\left(y_{1}, \ldots, y_{k}\right)$ in $H^{(k)}$. If $\mathfrak{A}$ has $\mathbb{A}_{1}$, so does $\mathcal{M}_{r}\left(\mathfrak{A}^{\left(r^{2}\right)}\right)$ (Proposition $2.6[\mathbf{9}]$ ), but the constants are not always good enough.

The infinite ampliation of any operator algebra has $\mathbb{A}_{1}(1)$. This is because weak* continuous functional on $\mathcal{B}(H)$ can be represented by a trace class operator. Using the polar decomposition, this can be realized as $\varphi=\sum_{i=1}^{\infty}\left[x_{i} y_{i}^{*}\right]$ where $\sum_{i}\left\|x_{i}\right\|^{2}=\sum_{i}\left\|y_{i}\right\|^{2}=\|\varphi\|$. So

$$
\varphi(T)=\sum_{i=1}^{\infty}\left\langle T x_{i}, y_{i}\right\rangle=\left\langle T^{(\infty)} \mathbf{x}, \mathbf{y}\right\rangle
$$

where $\mathbf{x}=\left(x_{1}, x_{2}, \ldots\right)$ and $\mathbf{y}=\left(y_{1}, y_{2}, \ldots\right)$ in $H^{(\infty)}$. In fact, this infinite ampliation is easily seen to have property $X_{0,1}$.

As in Theorem 3.4 , if $\mathcal{M}_{r}(\mathfrak{A})$ has property $\mathbb{A}_{1}(1)$, then we obtain an exact distance formula which yields a Nevanlinna-Pick type theorem for these algebras. The proof is the same.

Theorem 7.2. Suppose $\mathfrak{A}$ is a dual algebra of multipliers on $H$. If $\mathcal{M}_{r}(\mathfrak{A})$ has property $\mathbb{A}_{1}(1)$, then Lat $\mathfrak{A}$ is an $r \times r$ Nevanlinna-Pick family for $\mathfrak{A}$.

More generally, if the ampliation $\mathcal{M}_{r}\left(\mathfrak{A}^{(s)}\right)$ has $\mathbb{A}_{1}(1)$, then Lat $\left(\mathfrak{A}^{(s)}\right)$ is an $r \times r$ Nevanlinna-Pick family for $\mathfrak{A}$. In particular, Lat $\left(\mathfrak{A}^{(\infty)}\right)$ is a complete NevanlinnaPick family for any algebra of multipliers $\mathfrak{A}$.

While it appears that ampliations of matrix algebras over some well known multiplier algebras have $\mathbb{A}_{1}(1)$, we are unaware of any general results of this kind. Such a result would be interesting.

We will illustrate Theorem 7.2 with some examples.

Bergman Space. The Bergman shift $B$ on $A^{2}(\mathbb{D})$ has property $X_{0,1}[\mathbf{1 3}$. This is inherited by any dual subalgebra $\mathfrak{A}$ of $\mathfrak{M}\left(A^{2}(\mathbb{D})\right)$. Therefore $\mathcal{M}_{r}(\mathfrak{A})$ has property $\mathbb{A}_{1}$ (1) for all $r \geq 1$. We obtain a formulation of the complete Nevanlinna-Pick interpolation for subalgebras of $H^{\infty}$ in this context.

Theorem 7.3. Let $\mathfrak{A}$ be a dual subalgebra of $\mathfrak{M}\left(A^{2}(\mathbb{D})\right) \simeq H^{\infty}$. Let $E=\left\{z_{1}, \ldots, z_{n}\right\}$ be points in $\mathbb{D}$ which are separated by $\mathfrak{A}$, and let $W_{1}, \ldots, W_{n}$ be $r \times r$ matrices. There is an element $F \in \mathcal{M}_{r}(\mathfrak{A})$ with $F\left(z_{i}\right)=W_{i}$ and $\|F\| \leq 1$ if and only if the following 
holds: for each $L \in$ Lat $\mathfrak{A}$, (setting $N_{z_{i}}=L \ominus \mathfrak{J}_{z_{i}} L$ for $1 \leq i \leq n$ ), we have

$$
\left[\left(I_{r}-W_{i} W_{j}^{*}\right) \otimes P_{N_{z_{i}}} P_{N_{z_{j}}}\right]_{n \times n} \geq 0 \quad \text { for all } L \in \text { Lat } \mathfrak{A} \text {. }
$$

Proof. Let $A$ be any element of $\mathcal{M}_{r}(\mathfrak{A})$ such that $A\left(z_{i}\right)=W_{i}$. Since $\mathcal{M}_{r}(\mathfrak{A})$ has $\mathbb{A}_{1}(1)$, Theorem 3.3 implies that

$$
\operatorname{dist}\left(A, \mathcal{M}_{r}\left(\mathfrak{J}_{E}\right)\right)=\sup _{L \in \operatorname{Lat}(\mathfrak{A})}\left\|\left(P_{N_{L}} \otimes I_{r}\right) A\left(P_{N_{L}} \otimes I_{r}\right)\right\| .
$$

As before, a necessary and sufficient condition for interpolation with an element $F \in \mathcal{M}_{r}(\mathfrak{A})$ of norm at most one is that $\operatorname{dist}\left(A, \mathcal{M}_{r}\left(\mathfrak{J}_{E}\right)\right) \leq 1$. Also arguing in a standard manner, using the semi-invariance of $N_{L}$,

$$
\left\|\left(P_{N_{L}} \otimes I_{r}\right) A\left(P_{N_{L}} \otimes I_{r}\right)\right\|^{2}=\left\|\left(P_{N_{L}} \otimes I_{r}\right) A A^{*}\left(P_{N_{L}} \otimes I_{r}\right)\right\| .
$$

This has norm at most 1 if and only if

$$
\left(P_{N_{L}} \otimes I_{r}\right)\left(I-A A^{*}\right)\left(P_{N_{L}} \otimes I_{r}\right) \geq 0 .
$$

As we observed in Remark 2.9 $N_{L}$ is spanned by the spaces $N_{z_{i}}$ for $1 \leq i \leq n$. These subspaces are eigenspaces for $\left(\left.P_{L} \mathfrak{A}\right|_{L}\right)^{*}$, and thus they are independent, and at a positive angle to each other. So positivity of the operator above is equivalent to the positivity of

$$
\left[\left(P_{N_{z_{i}}} \otimes I_{r}\right)\left(I-A A^{*}\right)\left(P_{N_{z_{j}}} \otimes I_{r}\right)\right]=\left[\left(I_{r}-W_{i} W_{j}^{*}\right) \otimes P_{N_{\lambda_{i}}} P_{N_{\lambda_{j}}}\right]
$$

because the restriction of $\left(P_{L} \otimes I_{r}\right) A^{*}$ to $N_{z_{j}} \otimes \mathbb{C}^{r}$ is just $P_{N_{z_{j}}} \otimes W_{j}^{*}$.

Hardy Space. We return to the case of subalgebras of $H^{\infty}$ acting on Hardy space. In [14], for $\mathfrak{A}=H_{1}^{\infty}:=\left\{f \in H^{\infty}: f^{\prime}(0)=0\right\}$, it was shown that the distance formula for matrix interpolation fails for $\mathfrak{A}$. In our terminology, Lat $H_{1}^{\infty}$ is not a complete Nevanlinna-Pick family.

So we cannot drop the assumption that $\mathcal{M}_{r}(\mathfrak{A})$ has $\mathbb{A}_{1}(1)$. Indeed, the unilateral shift fails to have even property $\mathbb{A}_{2}$ (Theorem $3.7[\mathbf{8}]$ ). We will show that with ampliations, a general result can be obtained. The following result should be well known, but we do not have a reference. A version of it appears as Theorem 4 in [34].

Lemma 7.4. $\mathcal{M}_{r}\left(H^{\infty}\right)$ acting on $\left(H^{2} \otimes \mathbb{C}^{r}\right)^{(r)}$ as $r \times r$ matrices over $\mathfrak{M}\left(H^{2}\right)$ ampliated $r$ times has property $\mathbb{A}_{1}(1)$.

Proof. Form the infinite ampliation $\mathcal{M}_{r}\left(\mathfrak{M}\left(H^{2}\right)^{(\infty)}\right)$. Then any weak-* continuous functional $\varphi$ on $\mathcal{M}_{r}\left(H^{\infty}\right)$ with $\|\varphi\|<1$ can be represented as a rank one functional $\left[x y^{*}\right]$ on $\left(H^{2} \otimes \mathbb{C}^{r}\right)^{(\infty)} \simeq H^{2(\infty)} \otimes \mathbb{C}^{r}$ with $\|x\|\|y\|<1$. Write $x=\left(x_{1}, \ldots, x_{r}\right)$ and $y=\left(y_{1}, \ldots, y_{r}\right)$ with $x_{i}$ and $y_{i}$ in $H^{2(\infty)}$ so that if $F=\left[f_{i j}\right] \in \mathcal{M}_{r}\left(H^{\infty}\right)$, then

$$
\varphi(F)=\sum_{i, j=1}^{r}\left\langle M_{f_{i j}} x_{j}, y_{i}\right\rangle .
$$

Let $M=H^{\infty}\left[x_{1}, \ldots, x_{r}\right]$. By the Beurling-Lax-Halmos theory for shifts of infinite multiplicity [20], $\left.M_{z}^{(\infty)}\right|_{M}$ is unitarily equivalent to $M_{z}^{(s)}$ for some $s \leq r$. Thus we may assume that $x_{i}$ and $y_{j}$ live in $H^{2(r)}$. So this means that $x$ and $y$ are then identified with vectors in $\left(H^{2} \otimes \mathbb{C}^{r}\right)^{(r)}$ as desired.

We immediately obtain: 
Theorem 7.5. Suppose $\mathfrak{A}$ is a dual subalgebra of $H^{\infty}$ acting on $H^{2}$. Then $\operatorname{Lat}\left(\mathfrak{A}^{(r)}\right)$ is an $r \times r$ Nevanlinna-Pick family for $\mathfrak{A}$.

An additional application of the Beurling-Lax-Halmos result shows that Theorem 7.5 reduces to the matrix-valued Nevanlinna-Pick theorem when $\mathfrak{A}=H^{\infty}$.

In the case of $H_{1}^{\infty}$, this yields the result of Ball, Bolotnikov and Ter Horst [7. They express their models as invariant subspaces of $\mathcal{M}_{r}\left(H^{2}\right)$ (in the Hilbert Schmidt norm) instead of $H^{2(r)} \otimes \mathbb{C}^{r}$, but this is evidently the same space. It suffices to use subspaces which are cyclic for $H^{\infty}$. In much the same manner as [14, they obtain an explicit parameterization of these subspaces.

\section{REFERENCES}

[1] M.B. Abrahamse, The Pick interpolation theorem for finitely connected domains, Michigan Math. J. 26 (1979), 195-203.

[2] J. Agler and J.E. McCarthy, Complete Nevanlinna-Pick kernels, J. Funct. Anal. 175 (2000), 111-124.

[3] J. Agler and J.E. McCarthy, Pick interpolation and Hilbert function spaces, Graduate Studies in Mathematics 44, Amer. Math. Soc., Providence RI, 2002.

[4] C. Apostol, H. Bercovici, C. Foias, and C. Pearcy, Invariant subspaces, dilation theory, and the structure of the predual of a dual algebra. I, J. Funct. Anal. 63 (1985), 369-404.

[5] A. Arias and G. Popescu, Factorization and reflexivity on Fock spaces, Integral Equations Operator Theory 23 (1995),268-286.

[6] A. Arias and G. Popescu, Noncommutative interpolation and Poisson transforms, Israel J. Math. 115 (2000), 205-234.

[7] J. Ball, V. Bolotnikov, and S. ter Horst, A constrained Nevanlinna-Pick interpolation problem for matrix-valued functions, Indiana Univ. Math. J., to appear. arXiv:0809.2345 1

[8] H. Bercovici, C. Foias and C. Pearcy, Dilation theory and systems of simultaneous equations in the predual of an operator algebra, Michigan Math. J. 30 (1983), 335-354.

[9] H. Bercovici, C. Foias and C. Pearcy, Dual algebras with applications to invariant subspaces and dilation theory, CBMS Notes 56, Amer. Math. Soc., Providence RI, 1985.

[10] H. Bercovici, A note on disjoint invariant subspaces, Michigan Math. J. 34 (1987), 435-439.

[11] H. Bercovici, Factorization theorems and the structure of operators on Hilbert space, Ann. of Math. (2) 128 (1988), 399-413.

[12] H. Bercovici, Hyper-reflexivity and the factorization of linear functionals, J. Funct. Anal. 158 (1998), 242-252.

[13] Conway, J.B. and Ptak, M., The harmonic functional calculus and hyperreflexivity, Pacific J. Math. 204 No. 1 (2002).

[14] K.R. Davidson, V. Paulsen, M. Raghupathi and D. Singh, A constrained Nevanlinna-Pick interpolation problem, Indiana Univ. Math. J., 58 (2009), 709-732.

[15] K.R. Davidson and D.R. Pitts, Invariant subspaces and hyper-reflexivity for free semigroup algebras, Proc. London Math Soc 78 (1999), 401-430.

[16] K.R. Davidson and D.R. Pitts, The Algebraic structure of non-commutative analytic Toeplitz algebras, Math. Ann. 311 (1998), 275-303.

[17] K.R. Davidson and D.R. Pitts, Nevanlinna-Pick interpolation for non-commutative analytic Toeplitz algebras, Integral Eqtns. and Operator Theory 31 (1998), 321-337.

[18] S.I. Federov, V.L. Vinnikov, On the Nevanlinna-Pick interpolation in multiply connected domains, translation in J. Math. Sci. (New York), 105 (2001), 2109-2126.

[19] D.W. Hadwin and E.A. Nordgren, Subalgebras of reflexive algebras, J. Operator Theory 7 (1982), 3-23.

[20] P. Halmos, Shifts on Hilbert spaces, J. Reine Angew. Math. 208 (1961), 102-112.

[21] M. Kennedy, Wandering vectors and the reflexivity of free semigroup algebras, J. Reine Angew. Math., to appear (arXiv:0909.3479).

[22] M. Kennedy, Absolutely continuous representations of the non-commutative disk algebra, preprint arXiv:1001.3182

[23] J. Kraus and D. Larson, Reflexivity and distance formulae, Proc. London Math. Soc. 53 (1986), 340-356. 
[24] S. McCullough, Carathodory interpolation kernels, Integral Equations Operator Theory 15 (1992), 43-71.

[25] S. McCullough, The local de Branges-Rovnyak construction and complete Nevanlinna-Pick kernels, in "Algebraic Methods in Operator Theory", pp. 1524, Birkhauser, Basel, 1994.

[26] S. McCullough and V. Paulsen, $C^{*}$-envelopes and interpolation theory, Indiana Univ. Math. J., 51 (2002), no. 2, 479-505.

[27] V. Paulsen, Operator Algebras of Idempotents, J. Funct. Anal. 181 (2001), 209-226.

[28] V. Paulsen, An introduction to the theory of reproducing kernel Hilbert spaces, course notes, available at http://www.math.uh.edu/vern

[29] P. Quiggin, For which reproducing kernel Hilbert spaces is Pick's theorem true?, Integral Equations Operator Theory 16 (1993), 244-266.

[30] P. Quiggin, Generalisations of Pick's Theorem to Reproducing Kernel Hilbert Spaces, Ph.D. thesis, Lancaster University, 1994.

[31] M. Raghupathi, Nevanlinna-Pick interpolation for $\mathbb{C}+B H^{\infty}$, Integral Eqtns. and Operator Theory 63 (1) (2009), 103-125.

[32] M. Raghupathi, Abrahamse's interpolation theorem and Fuchsian groups, J. Math. Anal. Appl. 355 (1) (2009), 258-276.

[33] D. Sarason, The $H^{p}$ spaces of an annulus, Mem. Amer. Math. Soc. No. 56 (1965).

[34] D. Sarason, Generalized interpolation in $H^{\infty}$, Trans. Amer. Math. Soc. 127 (1967), 179-203.

[35] J. Thomson, Approximation in the mean by polynomials, Annals of Math. 133 (1991), 477507.

Pure Math. Dept., U. Waterloo, Waterloo, On N2L-3G1, CANadA

E-mail address: krdavids@uwaterloo.ca

Pure Math. Dept., U. Waterloo, Waterloo, On N2L-3G1, CANADA

E-mail address: rhamilto@math.uwaterloo.ca 